University of Nebraska - Lincoln

DigitalCommons@University of Nebraska - Lincoln

$9-2016$

\title{
Local Writing of Exchange Biased Domains in a Heterostructure of Co/Pd Pinned by Magnetoelectric Chromia
}

\author{
Uday Singh \\ University of Nebraska - Lincoln \\ William Echtenkamp \\ University of Nebraska-Lincoln, will.echtenkamp@huskers.unl.edu \\ M. Street \\ University of Nebraska - Lincoln \\ Christian Binek \\ University of Nebraska-Lincoln, cbinek@unl.edu \\ Shireen Adenwalla \\ University of Nebraska-Lincoln, sadenwalla1@unl.edu
}

Follow this and additional works at: https://digitalcommons.unl.edu/physicsadenwalla

Part of the Condensed Matter Physics Commons, Engineering Physics Commons, Materials Science and Engineering Commons, and the Nanoscience and Nanotechnology Commons

Singh, Uday; Echtenkamp, William; Street, M.; Binek, Christian; and Adenwalla, Shireen, "Local Writing of Exchange Biased Domains in a Heterostructure of Co/Pd Pinned by Magnetoelectric Chromia" (2016). Shireen Adenwalla Papers. 32.

https://digitalcommons.unl.edu/physicsadenwalla/32

This Article is brought to you for free and open access by the Research Papers in Physics and Astronomy at DigitalCommons@University of Nebraska - Lincoln. It has been accepted for inclusion in Shireen Adenwalla Papers by an authorized administrator of DigitalCommons@University of Nebraska - Lincoln. 


\title{
Local Writing of Exchange Biased Domains in a Heterostructure of Co/Pd Pinned by Magnetoelectric Chromia
}

\author{
Uday Singh, William Echtenkamp, M. Street, \\ Christian Binek, and Shireen Adenwalla
}

Department of Physics and Astronomy and Nebraska Center for Materials and Nanoscience, University of Nebraska-Lincoln, NE 68588, USA

Corresponding author —S. Adenwalla, email sadenwalla1@unl.edu

\begin{abstract}
The writing of micrometer-scaled exchange bias domains by local, laser heating of a thin-film heterostructure consisting of a perpendicular anisotropic ferromagnetic $\mathrm{Co} / \mathrm{Pd}$ multilayer and a (0001) oriented film of the magnetoelectric antiferromagnet $\mathrm{Cr}_{2} \mathrm{O}_{3}$ (chromia) is reported. Exchange coupling between chromia's boundary magnetization and the ferromagnet leads to perpendicular exchange bias. Focused scanning magneto-optical Kerr measurements are used to measure local hysteresis loops and create a map of the exchange bias distribution as a function of the local boundary magnetization imprinted in the antiferromagnetic pinning layer on field cooling. The robust boundary magnetization of the $\mathrm{Cr}_{2} \mathrm{O}_{3}$ fundamentally alters the exchange bias mechanism, enabling the writing of micrometer-scaled regions of oppositely directed exchange bias using a focused laser beam.
\end{abstract}

\section{Introduction}

Exchange bias (EB) ${ }^{[1,2]}$ refers to a shift in the magnetic hysteresis loop along the magnetic field axis and is most commonly seen in a ferromagnet (FM) in intimate contact with an antiferromagnet (AFM).

Published in Advanced Functional Materials 26 (2016), pp 7470-7478

DOI: $10.1002 /$ adfm.201602466

Copyright (c) 2016 WILEY-VCH Verlag GmbH \& Co. KGaA, Weinheim. Used by permission. Submitted May 18, 2016; revised July 29, 2016; published September 13, 2016. 
Exchange bias is extensively employed in magnetic memory devices, often to pin the magnetization of a ferromagnetic layer ${ }^{[3]}$ The pinning is reflected in a shift of the ferromagnetic hysteresis loop along the field axis. A phenomenological approach by Meiklejohn and Bean ${ }^{[4,5]}$ attributes this shift in the hysteresis loop to exchange coupling at the interface between the ferromagnet and antiferromagnet. In the simplest scenario, uncompensated spins at the AFM surface couple to the $F M$, resulting in an exchange bias field given by

$$
\mu_{0} H_{\mathrm{EB}}=-J \vec{S}_{\mathrm{AFM}} \cdot \vec{S}_{\mathrm{FM}} /\left(M_{\mathrm{FM}} t_{\mathrm{FM}}\right)
$$

where $S_{A F M}$ and $S_{F M}$ refer to the surface magnetization of the AFM and $\mathrm{FM}$, respectively, and $M_{\mathrm{FM}}$ and $t_{\mathrm{FM}}$ refer to the saturation magnetization and thickness of the FM. This approach greatly overestimates the magnitude of the $E B$, if we assume the interfacial coupling constant $J$ to be comparable to the coupling constant in magnetically ordered materials and further assume that all interface spins participate in the pinning. A large amount of work, both experimental and theoretical, $[2,6-8]$ has been devoted to an understanding of EB. In reality, the formation of antiferromagnetic domains, either lateral ${ }^{\left[{ }^{9}\right.} \mathrm{O}$ or through the depth of the film, ${ }^{[10]}$ will reduce the EB field. In addition, atomic level roughness and defects at the interface can have a significant impact on the density of uncompensated surface spins, also reducing the net exchange bias.

Here, we demonstrate laser written domains of EB in a heterostructure consisting of a $300 \mathrm{~nm}$ film of $\mathrm{Cr}_{2} \mathrm{O}_{3}(0001)$ and perpendicular anisotropic $\mathrm{Co} / \mathrm{Pd}$ multilayers. The spatial and temperature dependence of the EB will constrain the resolution of the domains that can be written. Careful measurements of the temperature dependence reveal a narrow distribution of blocking temperatures and spatially resolved measurements indicate that the spatial variations in EB are minimal. Both are facilitated by the robust roughness insensitive boundary magnetization ${ }^{[11,12]}$ of $\mathrm{Cr}_{2} \mathrm{O}_{3}$, a well-known magnetoelectric (ME) AFM insulator with a bulk Néel temperature of $307 \mathrm{~K}$. The boundary magnetization (BM) is present at the (0001) surface below the Néel temperature, enabled by the symmetry properties of magnetoelectric antiferromagnets ${ }^{[11-13]}$ and is coupled to the AFM order parameter. Below the Néel temperature, two equivalent AFM domain configurations (and corresponding boundary magnetizations) ${ }^{[11,12]}$ are 
possible. The roughness insensitive boundary magnetization fundamentally alters the EB mechanism-the statistical fluctuations seen in the Malozemoff model ${ }^{[14]}$ and the influence of defects do not play a role. Note that in our sample, the very large exchange bias that would be expected in the presence of this robust boundary magnetization is dramatically reduced due to the presence of the $5 \mathrm{~A} \mathrm{Pd}$ seed layer. We demonstrate local control of the EB using a focused laser beam and show that the ultimate size of these local regions may be manipulated by extrinsic controls, including the focusing optics, the power of the laser and the speed of writing. The process of domain writing involves the systematic control of sample temperature, the magnetization state of the FM layer, and the application of controlled localized heating provided by the focused laser beam. At a fundamental materials level, the unique boundary magnetization of $\mathrm{Cr}_{2} \mathrm{O}_{3}$ provides excellent intrinsic control over the exchange bias. We will show that the narrow distribution of blocking temperatures facilitates the ability to write well-defined patterns of exchange bias. Technologically, our work demonstrates the possibility of using heat-assisted magnetic recording (HAMR) to write a state variable by altering the $E B$, rather than the coercivity, of magnetic memory cells. The increased stability of EB coupled grains (as compared to uncoupled ferromagnets) and the possibility of reversing the EB with a small $(\approx 30 \mathrm{~K})$ thermal gradient, could lead to higher densities for magnetic recording.

The unique properties of magnetoelectric antiferromagnets and chromia, $\mathrm{Cr}_{2} \mathrm{O}_{3}$ in particular include the demonstrated isothermal electrically controlled exchange bias, an exciting phenomenon which could possibly lead to ultralow power spintronic applications. ${ }^{[13]}$ In bulk crystals of $\mathrm{Cr}_{2} \mathrm{O}_{3}$, magnetoelectric cooling (requiring both $E$ and $B$ fields) is necessary to produce a single-domain state, ${ }^{[12,13]}$ but in very thin films ${ }^{[15]}$ the Zeeman energy of the boundary magnetization makes it possible to produce a single-domain antiferromagnet using only B field cooling. Coupling a ferromagnet to the interface, however, allows for the production of a single-domain state in both bulk and thin film $\mathrm{Cr}_{2} \mathrm{O}_{3}$ if the $\mathrm{FM}$ is saturated on cooling through the Néel transition. We use both magnetoelectric annealing and the magnetization of the ferromagnet to control the EB. 


\section{Results}

Two samples are used in these experiments. Most of the measurements detailed here were carried out on a thin film heterostructure of sapphire $(0001) / \mathrm{Cr}_{2} \mathrm{O}_{3}(300 \mathrm{~nm}) / \mathrm{Pd}(0.5 \mathrm{~nm}) /[\mathrm{Co}(0.3 \mathrm{~nm}) / \mathrm{Pd}(1 \mathrm{~nm})]_{3}$ . The $\mathrm{Co} / \mathrm{Pd}$ multilayer with perpendicular magnetic anisotropy is exchange biased to the $\mathrm{Cr}_{2} \mathrm{O}_{3}$ film. Temperature-dependent measurements were performed both on the thin film heterostructure described above, as well as on a single crystal of $\mathrm{Cr}_{2} \mathrm{O}_{3}$, with a heterostructure of $\mathrm{Pd}(0.5 \mathrm{~nm}) /[\mathrm{Co}(0.3 \mathrm{~nm}) / \mathrm{Pd}(1 \mathrm{~nm})]_{3}$ grown on the single crystal (0001) $\mathrm{Cr}_{2} \mathrm{O}_{3}$ surface.

The crystal structure of the sample is important for the observation of EB because it is the (0001) surface of $\mathrm{Cr}_{2} \mathrm{O}_{3}$ that supports a roughness insensitive boundary magnetization that is exchange coupled to the AFM order parameter. X-ray diffraction measurements of the PLD grown $\mathrm{Cr}_{2} \mathrm{O}_{3}$ thin film, prior to the deposition of the $\mathrm{Co} / \mathrm{Pd}$ multilayers are shown in Figure 1a. Out-of-plane $\theta-2 \theta$ measurements (Figure 1a) indicate the presence of the necessary (0001) orientation. $\phi$-scans of (1014) peaks of both $\mathrm{Al}_{2} \mathrm{O}_{3}$ and $\mathrm{Cr}_{2} \mathrm{O}_{3^{\prime}}$ show identical threefold symmetry, indicating in-plane registry between the film and the substrate crystalline axes.

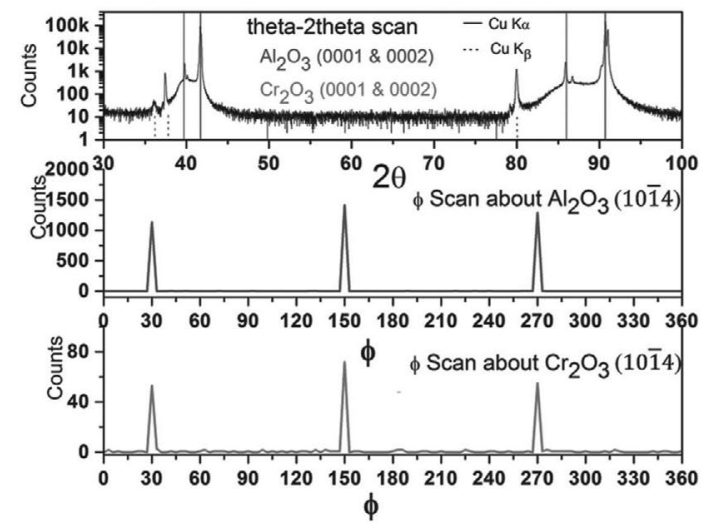

(a)

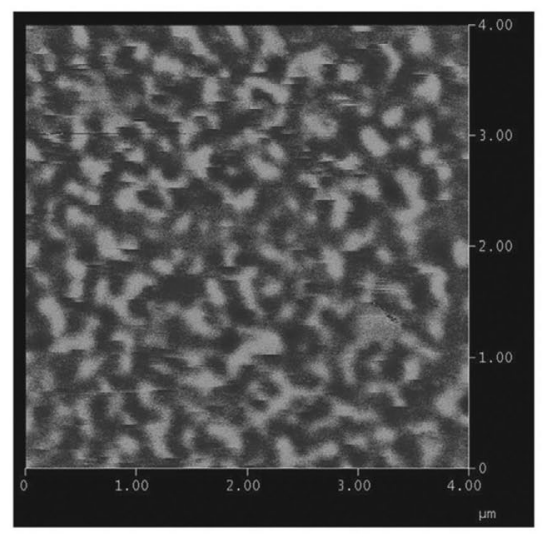

(b)

Figure 1. a) $\theta-2 \theta$ and $\phi X$-ray diffraction scans of $\mathrm{Cr}_{2} \mathrm{O}_{3}$ thin films grown on a sapphire (0001) substrate. The $\phi$-scan of the (1014) diffraction peak of both sapphire and $\mathrm{Cr}_{2} \mathrm{O}_{3}$ reveals the threefold symmetry in both materials, an indirect measure of the in-plane epitaxy. b) MFM image of magnetic domains at room temperature for the demagnetized film. 
We first investigate (a) lateral variations in the $E B$, created by varying the FM magnetization and then cooling through the Néel temperature of $\mathrm{Cr}_{2} \mathrm{O}_{3}$, and (b) the detailed temperature dependence to delineate the parameters for successful writing of EB domains. Ferromagnetic domains formed in the $\mathrm{Co} / \mathrm{Pd}$ will dictate the formation of AFM domains ${ }^{[16]}$ in $\mathrm{Cr}_{2} \mathrm{O}_{3}$ as well as the net averaged exchange bias field, $\mathrm{H}_{\mathrm{EB}}$. Domain sizes in $\mathrm{Co} / \mathrm{Pd}$ multilayers vary widely and are highly dependent on the thickness of the individual Co and Pd layers, the number of repeats, the seed layer, and the growth conditions. ${ }^{[17-25]}$ Magnetic force microscopy (MFM) scans (Figure 1b and Figure S3-1, Supporting Information) of our exchange biased sample in a demagnetized state shows the presence of stripe domains, a pattern characteristic of perpendicular anisotropy $\mathrm{Co} / \mathrm{Pt}$ and $\mathrm{Co} / \mathrm{Pd}$ multilayers system, with a median domain width $\approx 350 \mathrm{~nm}$. Careful analysis of the MFM data for demagnetized samples (Figure S3-2, Supporting Information) indicates domain areas ranging from 0.02 to $0.65 \mu \mathrm{m}^{2}$, with a median area of $0.12 \mu \mathrm{m}^{2}$. Domain sizes in $\mathrm{Cr}_{2} \mathrm{O}_{3}$ have not been investigated in detail, but XMCD-PEEM measurements indicate a domain size of $1-10 \mu \mathrm{m}^{2}$ in a virgin sample. ${ }^{12]}$ For domains that are smaller than the focused laser spot, focused MOKE measurements will provide the spatial average of the EB weighted by both the laser intensity profile and the spatial distribution of domains. Cooling at the saturation magnetization of the ferromagnet $\left(+M_{s}\right.$ and $\left.-M_{s}\right)$ will result in a single-domain AFM state, ${ }^{[26,27]}$ and the largest EB. Field cooling in multidomain states will result in a smaller integrated EB. ${ }^{[28]}$

\subsection{Exchange Bias Hysteresis Loops}

We start by measuring both microscopic and macroscopic hysteresis loops of nearly saturated samples. Figure $2 \mathrm{a}$ is a polar MOKE hysteresis loop acquired using focused MOKE with a beam spot of standard deviation $1.9 \mu \mathrm{m}$ at an arbitrary sample position, with $M / M_{\mathrm{s}}=-0.8$. The EB field was extracted by fitting the MOKE data to

$$
I(H)=\alpha+\beta^{\star} H+\gamma^{\star}\left(\operatorname{coth}\left(\delta^{\star}\left(H-H_{c}\right)\right)-1 / \delta^{\star}\left(H-H_{c}\right)\right)
$$

for both the forward and reverse field sweeps of the MOKE hysteresis loop. Equation ( 1) takes into account both the Faraday rotation from the objective lens and models the actual ferromagnetic hysteresis in 


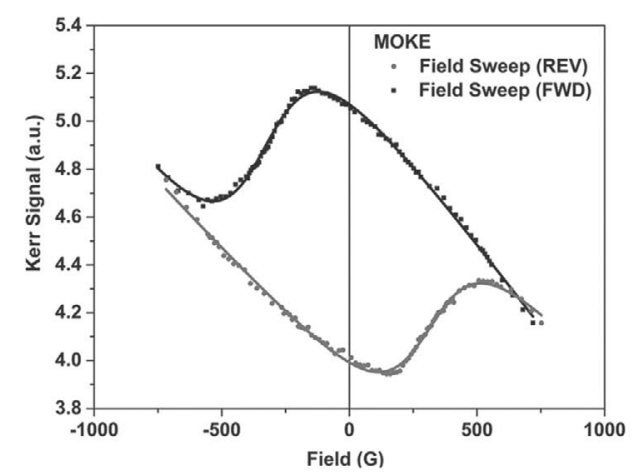

(a)

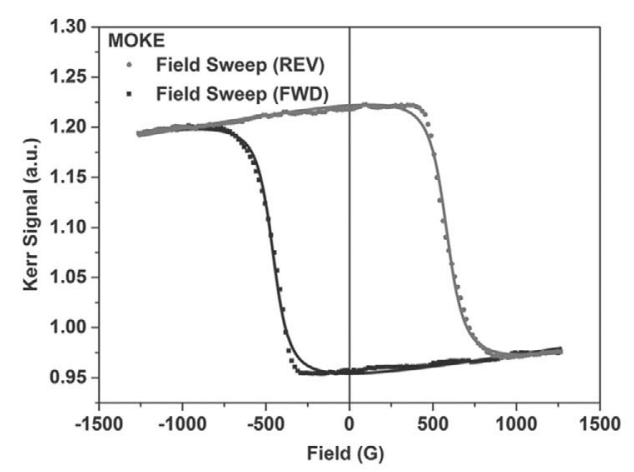

(b)

Figure 2. a) Microscopic (beam width $\sigma=1.9 \mu \mathrm{m}$ ) MOKE hysteresis loop obtained using focused polar MOKE at $T=270 \mathrm{~K}$, fit with Equation (1) for forward and reverse field sweeps. The sample was field cooled in negative magnetization (M/Ms $=-0.8$ ) from $T=314 \mathrm{~K}$. b) Macroscopic ( $1.5 \mathrm{~mm}$ beam diameter) MOKE loop together with the fit from Equation (1) at $T=240 \mathrm{~K}$. The sample was field cooled from $325 \mathrm{~K}$ in negative saturation $(\mathrm{M} / \mathrm{Ms}=-1)$.

terms of shifted Langevin magnetization curves. Although this is not a rigorous description of the hysteresis, it suffices as a phenomenological approximation to the data as indicated by the quality of the best fits (see Figure 2a,b).

The effects of field cooling and temperature for macroscopic and microscopic EB measurements are shown in Figure 3a,b. Cooling in positive saturation results in a negative value of $H_{E B}$, there is no signature in the coercive field (Figure $3 a, b$ ) at the blocking temperature and the hysteresis loops show similar behavior, with negligible

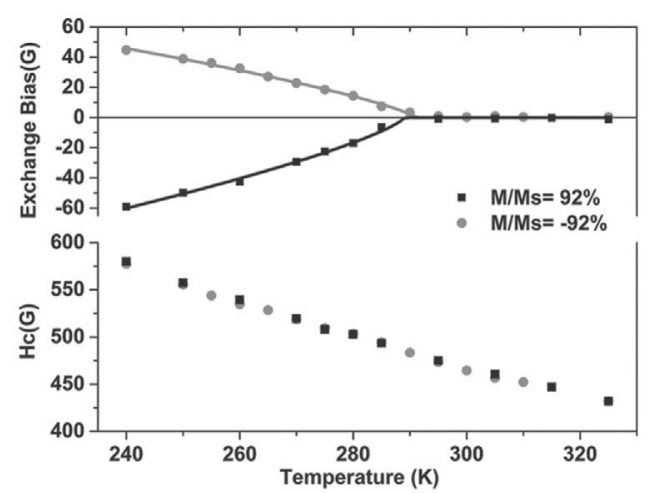

(a)

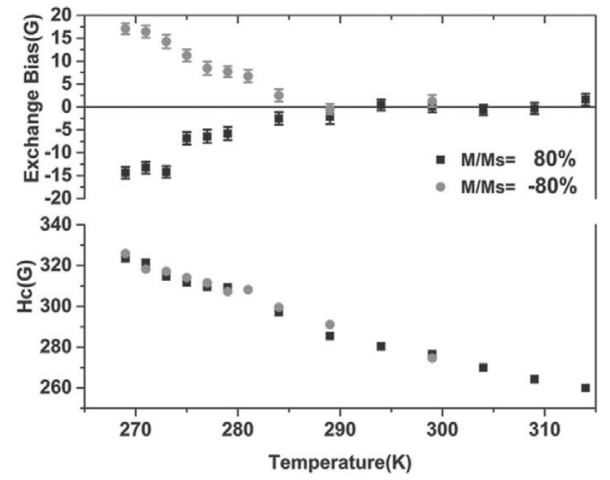

(b)

Figure 3. a) The macroscopic and b) local EB and coercive field as a function of temperature obtained with laser beam of diameter $1.5 \mathrm{~mm}$ and a focused laser beam of $\sigma=1.9 \mu \mathrm{m}$, respectively, for two different magnetization states of the FM layer. 
asymmetry, for increasing and decreasing fields, a strong indication that the reversal mechanisms are similar. The temperature dependence of the coercive field is at variance with the observed behavior of the coercive field in heterostructures of $\mathrm{Cr}_{2} \mathrm{O}_{3}$ and $\mathrm{Co} / \mathrm{Pt}$ multilayers [29,30] as well as in other more conventional systems, which show pronounced anomalies at $T_{N}$. In general, the enhanced coercivity in exchange biased samples is related to the presence of unpinned grains in the AFM, which are dragged along to rotate with the ferromagnet, thereby increasing the coercivity. ${ }^{[30]}$ The paucity of loose uncompensated spins at the chromia surface are consistent with the observed behavior of the coercive field.

\subsection{Distribution of Blocking Temperatures}

The ability to create micrometer-scaled laser-written EB patterns depends strongly on the distribution of local blocking temperatures. A wide distribution of blocking temperatures would degrade the ability to use localized heating as a precise patterning tool, for reasons we will discuss subsequently. In AFM materials with a variety of grain sizes and configurations, individual grains often have different coupling strengths and blocking temperatures. The conventional definition of the blocking temperature $\left(T_{B}\right)$ is the temperature at which the EB goes to zero, i.e., the maximum blocking temperature. However, because we are interested in the local $E B$, we need to determine the distribution of blocking temperatures, which is measured using the following protocol (sometimes called the York protocol ${ }^{[31]}$ ). The exchange biased sample is cooled from above the Néel temperature of the AFM in a positive field sufficient to saturate the ferromagnet, and then cooled to a measurement temperature $\left(T_{m}\right)$ below the AFM Néel temperature where the applied field direction is reversed. The sample temperature is raised and held at an activation temperature $\left(T_{\text {act }}\right)$, also below the Néel temperature, in the reversed field. The temperature is then lowered back to the measurement temperature $\left(T_{m}\right)$ and the EB of the sample is measured. This process is repeated for increasing activation temperatures and a graph of the EB as a function of activation temperature will go from positive to negative, through zero. The activation temperature at which $\mathrm{H}_{\mathrm{EB}}$ is zero is the median blocking temperature at which there is an equal distribution of oppositely directed antiferromagnetic domains resulting in a net zero EB. In a 
polycrystalline AFM system, this median blocking temperature $\left(T_{B}{ }^{\mathrm{YP}}\right)$ is always lower than the blocking temperature measured using conventional methods. ${ }^{[31,32]}$ The difference between the two temperatures and the width of the transition is a measure of the non-uniformity of the grain-by-grain coupling and can be significant in many thin film samples. For example, measurements on a variety of AFM/FM bilayers, including $\mathrm{CoFe} / \mathrm{/rMn}{ }^{[31]}$ and $\mathrm{FeNi} / \mathrm{NiO},{ }^{[33]}$ indicate widths greater than $100 \mathrm{~K}$, an effect attributed to interfacial disorder.

In contrast, both our samples show an extremely narrow distribution. ${ }^{[31]}$ We performed measurements on both the bulk single crystal of $\mathrm{Cr}_{2} \mathrm{O}_{3}$ with multilayers of [Co/Pd] identical to the thin film sample, as well as on the thin film sample. Measurements ${ }^{\left[{ }^{11]}\right.}$ on the bulk single crystal sample indicate a median blocking temperature $\left(T_{B}{ }^{\mathrm{YP}}\right)$ of $309.1 \pm 0.02 \mathrm{~K}$ with a vanishingly small width, well below $0.5 \mathrm{~K}$. As described in the Introduction, bulk crystals require magnetoelectric annealing to ensure a single domain. Cooling from 350 to $270 \mathrm{~K}$ in $E$ $=+100 \mathrm{~V} \mathrm{~mm}^{-1}$ and $B=+70 \mathrm{mT}$ resulted in a positive EB at $270 \mathrm{~K}$. After the measurement, the sample temperature was raised to an activation temperature $\left(T_{\text {act }}\right)$ in a reversed magneto-electric field $(E=-1050$ $V \mathrm{~mm}^{-1}$ and $B=+240 \mathrm{mT}$ ), which facilitates negative $E B$ in the system. The sample was cooled back to $270 \mathrm{~K}$ and the measured EB field as a function of activation temperature is shown in Figure 4a. Fitting this to a log-normal cumulative distribution function (CDF), ${ }^{[34]}$ and taking a scaled derivative of the fitted CDF gives the log-normal probability density function (PDF). The PDF is a measure of the distribution of blocking temperatures which is proportional to the switching probability of AFM domains at a given activation temperature. ${ }^{[3,35-37]}$ The data indicate an extremely narrow distribution of blocking temperatures in the single crystal $\mathrm{Cr}_{2} \mathrm{O}_{3}$ sample, with a median blocking temperature of $309.1 \pm 0.02 \mathrm{~K}$ (slightly above the reported values of $T_{\mathrm{N}}$ ${ }^{[13]}$ ) and a width of less than $0.5 \mathrm{~K}$. The small discrepancy may be related to the presence of loose spins above $T_{\mathrm{N}}$ and/or a temperature lag while ramping to $T_{\text {act }}$.

We performed similar measurements on the thin film $\mathrm{Cr}_{2} \mathrm{O}_{3}-\mathrm{Co} / \mathrm{Pd}$ heterostructures, but rather than macroscopic hysteresis loops, we took line scans with a step size of $1 \mu \mathrm{m}$ to look for local variations. The thin film was initially field cooled from 320 to $270 \mathrm{~K}$ in $M / M_{s=}-0.8$ followed by reversal of the magnetization $\left(M / M_{s}=0.8\right)$. Hysteresis loops were measured along the line at $270 \mathrm{~K}$ and the line scans of the $\mathrm{EB}$ 

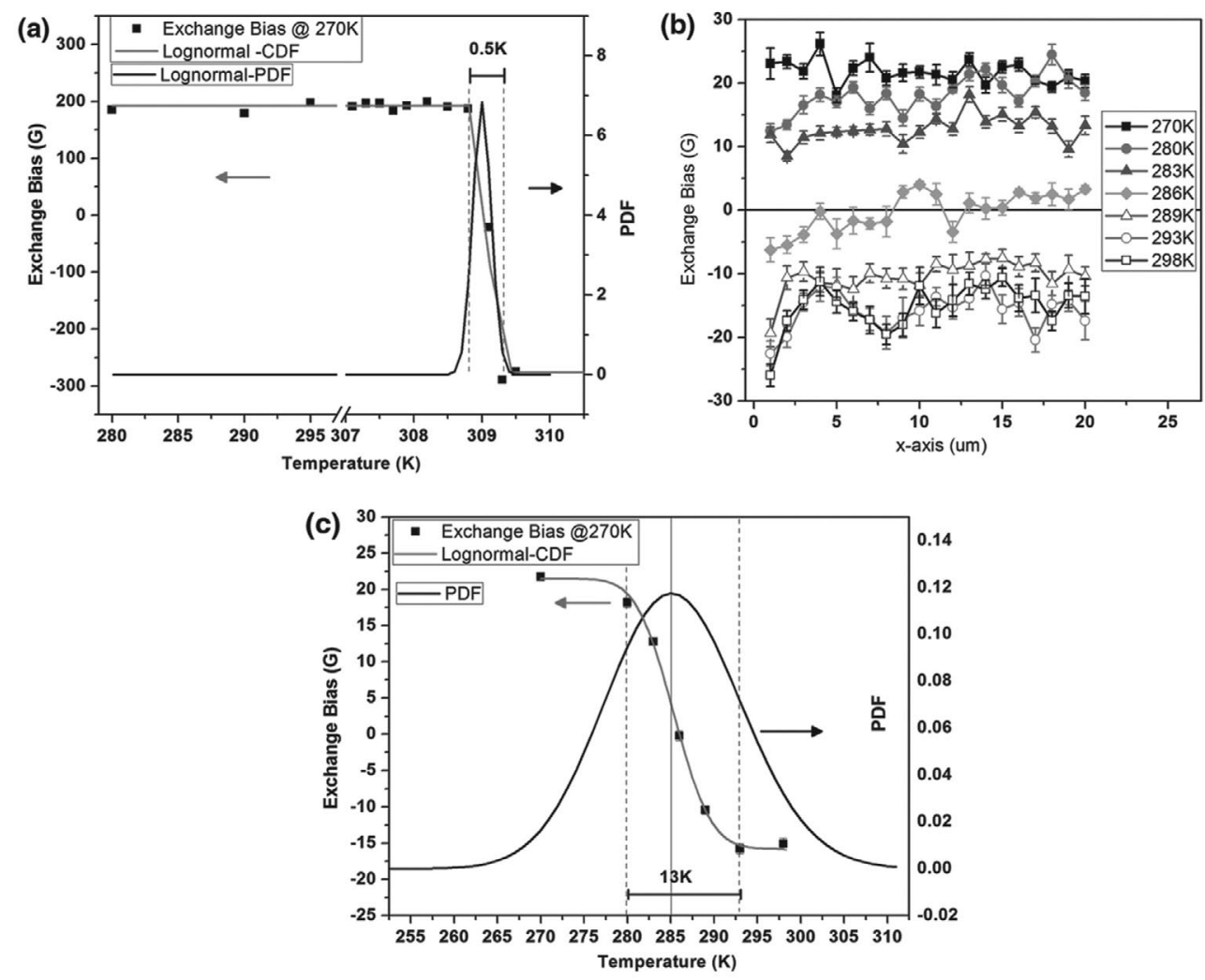

Figure 4. a) Macroscopic EB versus activation temperature $\left(T_{\text {act }}\right)$ for $\mathrm{Co} / \mathrm{Pd}$ multilayers on $\mathrm{C}_{2} \mathrm{O}_{3}$ single crystal (the error bars are smaller than the symbol size). The data are fit to a log-normal cumulative distribution function (CDF) (gray) and the black line is the scaled derivative of the CDF. The configuration of the multilayer stack is identical to that of the thin film sample. b) Microscopic EB measured along a line of width $2.0 \mu \mathrm{m}$ at $270 \mathrm{~K}$ for the thin film $\mathrm{Cr}_{2} \mathrm{O}_{3}$ heterostructure as a function of activation temperature $\left(T_{\text {act }}\right)$ after initial cooling in negative magnetization $(M / M s=-0.8)$. $c)$ The plot of the line average of EB versus activation temperatures $\left(T_{\text {act }}\right)$ for the thin film heterostructure, once again the error bars are smaller than the symbols. The data are fit to a log-normal CDF. The black line is the scaled derivative of the CDF.

at a variety of activation temperatures are shown in Figure $4 \mathrm{~b}$, exhibiting variations of $\approx 10 \mathrm{G}$ at all activation temperatures. There are no regions of oppositely directed EB except at $286 \mathrm{~K}$, the median blocking temperature. The line averaged EB is shown in Figure 4c. We obtain the PDF in a similar manner as described above, finding a median blocking temperature $\left(T_{B}^{\mathrm{YP}}\right)$ of $285.3 \pm 0.2 \mathrm{~K}$ with a width of $\approx 13$ $\mathrm{K}$ for the thin film samples. This may be compared to the conventional measurement, with a $T_{B}(\max )$ of $290 \pm 1 \mathrm{~K}$, a difference of only $4 \mathrm{~K}$. Because $T_{B}{ }^{\mathrm{YP}}$ and $T_{B}$ (max) (as measured by more conventional 
measurements) agree to within $4 \mathrm{~K}$ and because the width of the transition is so narrow compared to other AFM/FM bilayers with widths

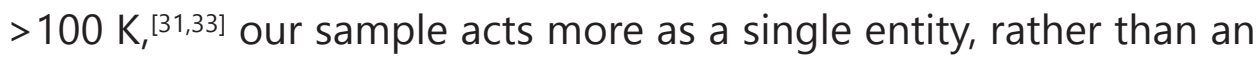
ensemble of small, independent AFM grains. ${ }^{[31,33,35-37]}$ As we shall see, both the spatial uniformity and the narrow temperature distribution are important prerequisites for laser writing.

\subsection{Spatial Variations in Exchange Bias}

The spatial variation in the EB will determine the limiting size of the exchange biased regions that we can write. In heat-assisted magnetic recording media, ${ }^{[38]}$ exchange biased films are used to stabilize nanomagnets against the superparamagnetic limit and to increase the coercive field and effective magnetic anisotropy energy. ${ }^{[39]}$ The use of EB in such applications requires an understanding of the spatial dependence and methods for local manipulation of magnetic properties at scales well below the wavelength of light. ${ }^{[38]}$ Among conventional exchange biased systems, there are very few studies that investigate the spatial dependence and control of EB. An elegant XMCD-PEEM experiment on a virgin $\mathrm{Co} / \mathrm{LaFeO}_{3}$ bilayer ${ }^{[40]}$ with numerous lateral domains of varying size indicate that the EB in these systems scales inversely as the area of the domains, as would be expected if EB arises from the statistical nature of atomic level roughness. Experiments on bilayers of $\mathrm{Ni} / \mathrm{FeF}_{2}{ }^{[41]}$ have probed the spatial resolution, albeit at very coarse length scales of the order of $1 \mathrm{~mm}$ to investigate the modes of reversal of different lateral domains.

We performed micrometer-scaled measurements of $\mathrm{H}_{\mathrm{EB}}$, over a 25 $\mu \mathrm{m} \times 25 \mu \mathrm{m}$ region for four different field cooling conditions-with $M / M_{s}=+0.8$ and -0.8 , zero field cooling of a demagnetized sample and finally field cooling at $H_{c}$, where the magnetization of the Co/Pd is also zero. Local hysteresis loops were acquired at a step size of 2.5 $\mu \mathrm{m}$ using a focused He-Ne laser beam spot with a standard deviation of $1.9 \mu \mathrm{m}$. The hysteresis loops were fit with Equation (1), from which we mapped $\mathrm{H}_{\mathrm{EB}}$.

Figure 5a are EB maps at $268 \mathrm{~K}$ for the different field cooling conditions (also see Figure S4-1, Supporting Information). For field cooling at $M / M_{s}=+0.8\left(M / M_{s}=-0.8\right)$, the sign of the EB field is negative (positive) over the entire region, with a magnitude ranging from -10 to $-22 \mathrm{G}(+10$ to $+22 \mathrm{G})$. MFM measurements (Figure S3-3, Supporting 

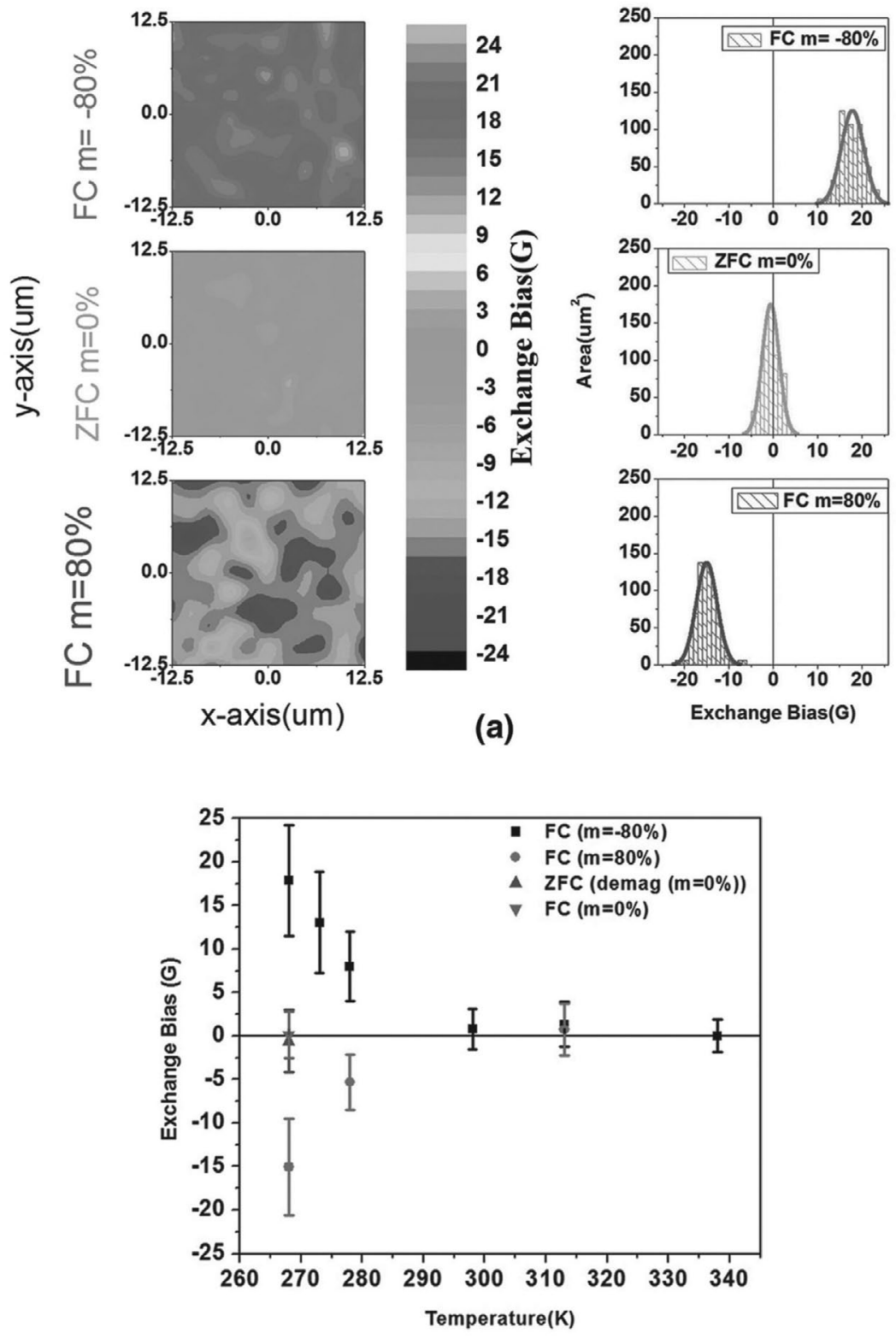

(b)

Figure 5. a) Spatial EB maps (left) and a histogram (right) of the $E B$ at $268 \mathrm{~K}$ for field cooling in $M / M s=-0.8$, zero remanent magnetization in zero field and $M / M s=0.8$. The histogram is binned in steps of $1 \mathrm{G}$ and fit to a normal distribution. b) The temperature dependence of the mean value and the variance (indicated by the error bar) of EB obtained from the histograms.

Information) show that over the microscopic areas probed by the focused laser beam, the magnetization ranges from 0.6 to $0.9 \mathrm{M}_{\mathrm{s}}$, accounting for the range of $\mathrm{H}_{E B}$. 
The EB maps for field cooling in zero net magnetization (either zero field cooling of a demagnetized sample or field cooling at $H_{c}$ ), (Figure 5b and Figure S4-1(h), Supporting Information) show local variations, but with an average value of nearly zero, and are very similar to the map at $338 \mathrm{~K}$, well above the Néel temperature, for $M / M_{s}=-0.8$ (Figure S4-1(i), Supporting Information).

The variation in the EB fields with FM layer magnetization and temperature is apparent in histogram plots shown in Figure 5a, and Figures S4-2 and S4-3 in the Supporting Information. The $x$-axis is binned in steps of $1 \mathrm{G}$ and the $y$-axis represents the total area with that value of EB. Both the mean EB and the variance of the distribution are obtained by fitting the histogram to a normal distribution (solid line) and are summarized in Figure 5b with the mean and the variance represented by solid symbols and error bars, respectively. The mean values of the EB for $M / M_{s}=0.8(-0.8),-15 G(17 \mathrm{G})$ are comparable to the corresponding macroscopic values shown in Figure 3a. The variance in the EB field is larger at low temperature for non-zero magnetization $\left(M / M_{s}= \pm 0.8\right)$ and decreases with temperature. Above the blocking temperature, the variance is equal to the instrumental error. MFM measurements of magnetic domains have been performed as a function of field and analysis of the domain size and distribution (see Section S-3 and Figures S3-2 and S3-3 in the Supporting Information) indicate that the major contributor to the variance in EB is the variance in the magnetization. For $M / M_{s}= \pm 0.8$, for example, Figure S3-3 in the Supporting Information indicates that the magnetization on a microscopic length scale can vary between 0.6 and $0.9 M_{S}$, resulting in variations in the EB.

\subsection{Localized Control of Exchange Bias}

The well-defined temperature dependence and the absence of significant local areas of reversed EB allow for the tailored writing of regions of EB. We first "write" EB using localized magnetic fields, at a fairly coarse scale, to demonstrate the effect of local magnetization control of the ferromagnet during cooling. We saturate the sample and then create a local variation in the magnetization by energizing a small electromagnet with a $100 \mu \mathrm{m}$ diameter pole piece, thereby generating a spatially confined oppositely directed magnetic field. A line scan of the Kerr signal across this region ( Figure 6 ) (beam width 


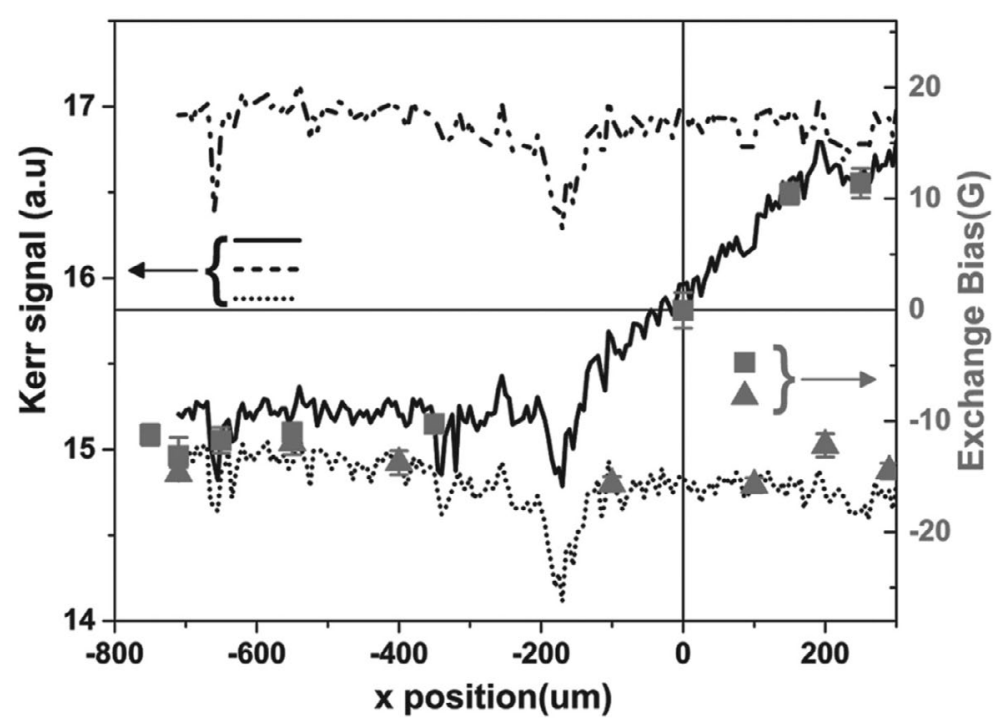

Figure 6. Demonstration of local control of EB by local variation of the magnetization state during cooling. Left axis indicates magnetization in arbitrary units and the right axis indicates the EB, both as functions of position. The dot, dashed and solid black lines are line scans of the magnetization (Kerr signal) for positive, negative and spatially varying magnetization, respectively. (The prominent feature appearing in all three lines at $x=-190 \mu \mathrm{m}$ is due to a local defect and is not relevant). The symbols are values of the EB at discrete intervals along the line, at $T=268 \mathrm{~K}$. The triangles indicate $\mathrm{H}_{\mathrm{EB}}$ for the positive saturation state of the $\mathrm{FM}$ (represented by the dotted lines). The squares indicate $\mathrm{H}_{E B}$ for the spatially varying magnetization state represented by the black solid line and is a clear indication that the EB in the system follows the magnetization profile.

$\sigma=0.8 \mu \mathrm{m}$ ) shows a monotonically varying magnetization (thin black line) varying from $\approx+0.75 M_{S}$ to $\approx-0.75 M_{S}$ (looking from left to right) over a distance of $1 \mathrm{~mm}$. The dotted and dashed lines in Figure 6 are the Kerr signals for positive and negative field saturation, respectively, and set the scale of the magnetization. Although the smaller signal for positive saturation seems somewhat confusing, note that the Kerr signal depends on the relative angle between the polarizer and analyzer and that the sign of $M_{s}$ (whether positive or negative) is obtained from hysteresis loops. On cooling to $268 \mathrm{~K}$, the locally measured EB, indicated by the solid symbols, track the magnetization profile, albeit with a reversed sign as expected.

Our results indicate that local control of the ferromagnetic magnetization allows for control of AFM domains and hence local EB. We achieve local modulation of the EB on a microscopic scale via a 
protocol involving temperature, the magnetization of the FM layer and the heat supplied by a focused laser beam. Figure $7 a$ is a flow chart of the process for EB writing. The process is initialized by cooling the sample in a fully magnetized FM state from above the Néel temperature to a temperature $\left(T_{R}\right)$ below the blocking temperature, resulting in a single AFM domain. ${ }^{[26,27]}$ At $T_{R^{\prime}}$ the magnetization direction of the FM layer is flipped and the sample temperature is increased to a writing temperature $\left(T_{w}\right)$ below the median blocking temperature. The choice of writing temperature is crucial, as the York protocol measurements indicate. A temperature just below the median blocking temperature, $T_{B}^{\mathrm{YP}}$, rather than $T_{B}$ ensures the stability of the AFM domains and once again highlights the importance of the relatively narrow distribution

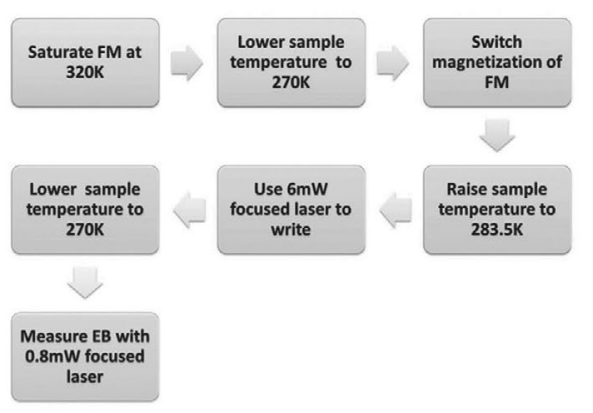

(a)

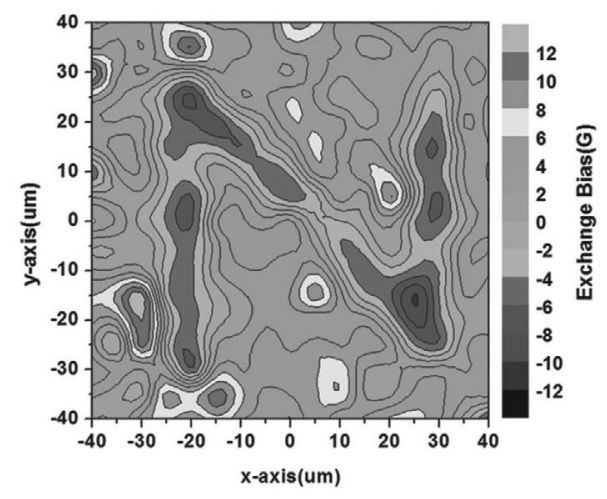

(c)

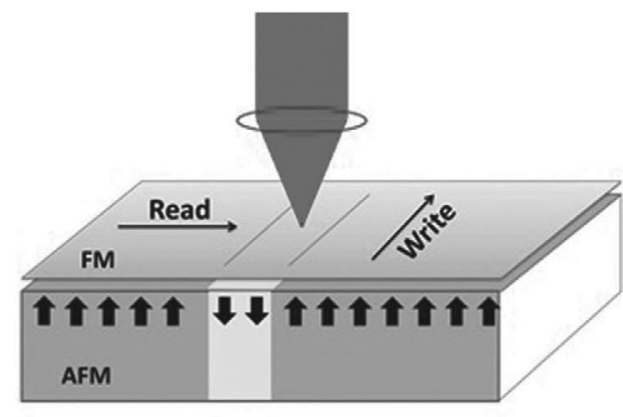

(b)

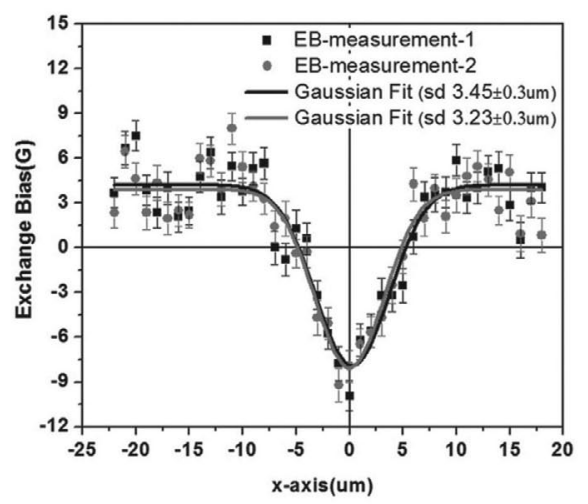

(d)

Figure 7. a) Flow chart showing the process for local modulation of the EB in the $\mathrm{Cr}_{2} \mathrm{O}_{3}-\mathrm{Co} / \mathrm{Pd}$ multilayer thin film. b) A sketch representing the change in boundary magnetization of the AFM with localized heating during writing with the laser beam writing. c) An EB map after laser writing of the letter " $N$ ". d) The EB bias along the read line (perpendicular to the write line), showing the spatial profile of the written EB fitted to a Gaussian profile. A laser beam width of $\sigma=1.9 \mu \mathrm{m}$ was used to write and read the EB. 
of blocking temperatures. With the sample held at $T_{W}=283.5 \mathrm{~K}$, a focused laser beam is used to locally heat the sample to above the Néel temperature. Figure $7 \mathrm{~b}$ sketches the expected changes in the boundary magnetization of $\mathrm{Cr}_{2} \mathrm{O}_{3}$ after EB writing with a focused laser beam. The exchange coupling alters the spin configuration within the AFM to adapt to the magnetization state of the FM as it is cooled back below the Néel temperature. Figure 7c demonstrates our ability to write $E B$ patterns, showing a map of the EB, after laser writing. The laser written region (" $\mathrm{N}$ ") displays EB of the opposite sign.

We investigate the dependence of this region of oppositely directed EB (and by inference, of oppositely directed boundary magnetization), on both the laser beam width and the writing speed. Two different laser beam widths were used for writing, one with a beam width of $\sigma=$ $1.9 \mu \mathrm{m}$ and one with $\sigma=0.8 \mu \mathrm{m}$, both with identical laser powers of $\approx 6$ $\mathrm{mW}$. The Gaussian laser beam generates a Gaussian temperature profile on the sample surface, with a broadening due to thermal diffusivity.

We first wrote a line of EB at $T_{W}=283.5 \mathrm{~K}$ using the broader laser beam with a beam width of $\sigma=1.9 \mu \mathrm{m}$. The beam was scanned over the sample in discrete steps of $1 \mu \mathrm{m}$, pausing for two seconds between steps. The EB was measured at $270 \mathrm{~K}$ using a much lower power, $0.8 \mathrm{~mW}$, focused laser along a read line normal to the write direction, and the result is shown in Figure $7 \mathrm{~d}$. The written EB along the read line was measured twice and both measurements can be fitted to identical Gaussian profiles with equal standard deviations of $3.3 \mu \mathrm{m}$. This reproducibility implies the reading process does not modify the EB. The EB standard deviation is almost $60 \%$ larger than the write laser beam width $(\sigma=1.9 \mu \mathrm{m})$, an effect we attribute to thermal diffusion.

We repeated the process of writing and reading with the more tightly focused laser beam, of beam width $\sigma=0.8 \mu \mathrm{m}$, at two different writing speeds. The laser intensity was modulated (at $1201 \mathrm{~Hz}$ ) and the beam advances in $1 \mu \mathrm{m}$ steps with delays of 2 and $0.4 \mathrm{~s}$, respectively, corresponding to average velocities of 0.5 and $2.5 \mu \mathrm{m} \mathrm{s}^{-1}$. The EB profile and the Gaussian fit to the profile for the two writing speeds is shown in Figure 8 a indicating that the effects of the fivefold increase in laser beam speed reduces the width by nearly $40 \%$, while maintaining an identical height. A similar reduction in the width of the temperature profile was observed in a simulation of the temperature distribution, as shown in Figure 8b. (Detailed information on the simulation is presented in the Supporting Information). 


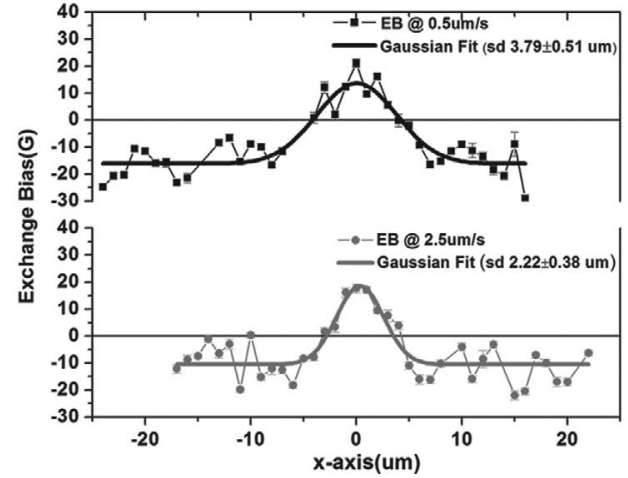

(a)

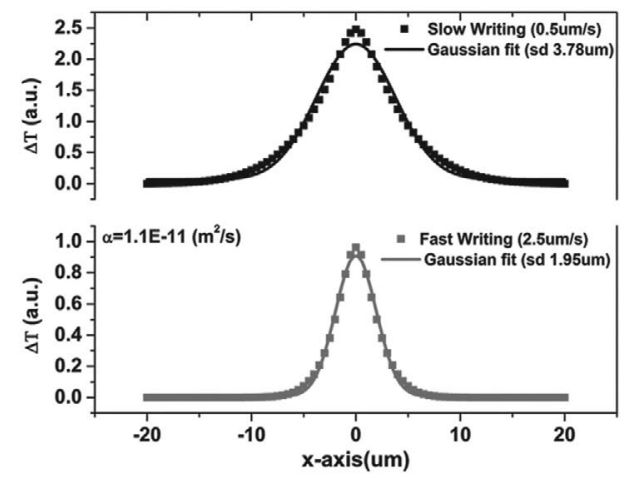

(b)

Figure 8. a) EB profile written using a Gaussian laser beam with a standard deviation of $0.8 \mu \mathrm{m}$ at two speeds, $0.5 \mu \mathrm{m} \mathrm{s}^{-1}$ (black) and $2.5 \mu \mathrm{m} \mathrm{s}^{-1}$ (gray). The faster writing speed results in a $40 \%$ reduction in the width of the reversed EB region. b) Simulation of the temperature profile generated by the focused laser beam for 0.5 and $2.5 \mu \mathrm{m} \mathrm{s}^{-1}$ writing speeds.

\section{Conclusion}

In conclusion, we have demonstrated the ability to write exchange bias domains using a scanning laser beam in a $\mathrm{Cr}_{2} \mathrm{O}_{3}-\mathrm{Co} / \mathrm{Pd}$ exchange coupled system. Measurements on the size and shape of the written domains indicate that excellent extrinsic control is possible by varying the focal spot size, the power and the speed of the write beam. This ability is enabled by the robust boundary magnetization of magnetoelectric $\mathrm{Cr}_{2} \mathrm{O}_{3}$, which in turn results in a very narrow distribution of blocking temperatures. A wider distribution of blocking temperatures would necessitate a lower write temperature (to ensure only one type of domain) and a correspondingly higher power of the writing laser, resulting in far poorer spatial resolution, due to thermal diffusion effects. Clearly, an understanding of the material properties of $\mathrm{Cr}_{2} \mathrm{O}_{3}$ is of fundamental importance to this unique ability to write exchange bias domains, because exchange with the stable and roughness insensitive boundary magnetization (rather than with the spatially fluctuating uncompensated spins at the surface of a typical antiferromagnet) leads to the observed spatial and temperature dependence of the exchange bias.

Our experiments on laser writing are within the diffraction limit. For future applications, however, experiments on near-field transducers using surface plasmons ${ }^{[42]}$ show that write beams well below the 
diffraction limit, ( $\approx 70 \mathrm{~nm}$ wide) may be used for laser writing and similar near field transducers have already been incorporated into read heads. The use of $\mathrm{Cr}_{2} \mathrm{O}_{3}$ based heterostructures will be particularly advantageous for heat-assisted magnetic recording applications because of the small local temperature gradient that is necessary to write exchange bias domains of opposite sign. The narrow temperature distribution indicates that a local temperature differential of $30-50 \mathrm{~K}$ is enough to switch the exchange bias, as compared to approximately $300 \mathrm{~K}{ }^{[38]}$ used for conventional heat assisted magnet recording, which lowers the coercive field of a ferromagnet. Other possible extrinsic control mechanisms that remain to be explored include beam modulation, controlling for heat diffusion/absorption by choice of materials and variations of the writing temperature. Our experiments on the local manipulation of exchange bias and domains in the coupled antiferromagnet open a pathway to easily rewritable domains in technologically important materials.

\section{Experimental Section}

Two samples are used in these experiments. The thin film heterostructure of Sapphire $(0001) / \mathrm{Cr}_{2} \mathrm{O}_{3}(300 \mathrm{~nm}) / \mathrm{Pd}(0.5 \mathrm{~nm}) /[\mathrm{Co}(0.3 \mathrm{~nm}) /$ $\mathrm{Pd}(1 \mathrm{~nm})]_{3}$ was grown as follows. The $\mathrm{Cr}_{2} \mathrm{O}_{3}$ film, was grown on a (0001) single crystal sapphire substrate using pulsed laser deposition (PLD). Subsequently, a $0.5 \mathrm{~nm}$ thick Pd seed layer and [Co(0.3 nm)/ $\mathrm{Pd}(1 \mathrm{~nm})]_{3}$ multilayers were deposited using molecular beam epitaxy (MBE). The role of the $0.5 \mathrm{~nm}$ Pd seed layer is to inhibit the formation of $\mathrm{CoO}$ at the $\mathrm{Cr}_{2} \mathrm{O}_{3}$ interface, to promote the perpendicular anisotropy of the Co/Pd multilayer and to tune the strength of the EB field and coercivity. ${ }^{[43,44]}$ Detailed information regarding deposition conditions is provided in Section S1 in the Supporting Information. Details on the growth conditions of the heterostructure on the single crystal $\mathrm{Cr}_{2} \mathrm{O}_{3}$ are provided in He et al. [13]

Macroscopic exchange bias measurements were obtained using the magneto-optical Kerr effect (MOKE) with a laser beam of diameter $1.5 \mathrm{~mm}$. Focused scanning MOKE was used to measure local hysteresis loops and create a map of the EB distribution. Two focused MOKE setups were employed, one with an $\mathrm{He}-\mathrm{Ne}(632 \mathrm{~nm} 10 \mathrm{~mW})$ laser focused using a $20 \times$ objective lens (with numerical aperture (NA) 
of 0.40 ) to a Gaussian beam spot of width (standard deviation) $\sigma=$ $1.9 \mu \mathrm{m}$ (FWHM $4.5 \mu \mathrm{m}$ ), and the second a $658 \mathrm{~nm}$ diode laser (Hitachi HL6501) focused using a $60 \times$ objective (NA 0.75) to a Gaussian beam spot of width $\sigma=0.8 \mu \mathrm{m}$ (FWHM $1.8 \mu \mathrm{m}$ ). In the latter, more focused, MOKE geometry the laser beam intensity was modulated at $1201 \mathrm{~Hz}$ to improve the signal-to-noise ratio. Detailed information regarding focused MOKE is provided in Section S2 in the Supporting Information and in Singh and Adenwalla. ${ }^{[45,46]}$ The exchange biased sample was mounted on an aluminum sample stage equipped with a thermoelectric cooler and a thermocouple for temperature control and an electromagnet with a $2 \mathrm{~mm}$ diameter pole piece. Running the magnet at full power for extended periods of time led to sample heating; hence most microscopic EB measurements were performed after saturating the sample, turning the field off and cooling though the blocking temperature, so that the ferromagnet was in its remanent state with $M=0.8 \mathrm{Ms}$. The thermoelectric cooler and electromagnet were driven by two separate Kepco bipolar power supplies controlled via Labview. The entire sample unit (sample, cooler, thermocouple, and magnet) was positioned on a micro-positioning stage driven by a DC servo motor using a Newport ESP-300 controller with a positional accuracy of $0.2 \mu \mathrm{m}$.

Supporting Information follows the References.

Acknowledgements - This work was supported by the National Science Foundation (NSF) through grant No. DMR-1409622 and the Nebraska Materials Research Science and Engineering Center (MRSEC) (grant No. DMR-1420645). The research was performed in part in the Nebraska Nanoscale Facility: National Nanotechnology Coordinated Infrastructure and the Nebraska Center for Materials and Nanoscience, which are supported by the National Science Foundation under Award ECCS: 1542182 and the Nebraska Research Initiative.

\section{References}

[1] A. E. Berkowitz, K. Takano, J. Magn. Magn. Mater. 1999, 200, 552.

[2] J. Nogués, I. K. Schuller, J. Magn. Magn. Mater. 1999, 192, 203.

[3] C. Y. You, H. S. Goripatti, T. Furubayashi, Y. K. Takahashi, K. Hono, Appl. Phys. Lett. 2008, 93, 012501.

[4] W. H. Meiklejohn, C. P. Bean, Phys. Rev. 1956, 102, 1413.

[5] W. H. Meiklejohn, C. P. Bean, Phys. Rev. 1957, 105, 904.

[6] M. Kiwi, J. Magn. Magn. Mater. 2001, 234, 584. 
[7] P. K. Manna, S. M. Yusuf, Phys. Rep. 2014, 535, 61.

[8] E. V. Gomonay, V. M. Loktev, Low Temp. Phys. 2014, 40, 17.

[9] D. Spanke, V. Solinus, D. Knabben, F. U. Hillebrecht, F. Ciccacci, L. Gregoratti, M. Marsi, Phys. Rev. B 1998, 58, 5201.

[10] M. D. Stiles, R. D. McMichael, Phys. Rev. B 1999, 59, 3722 .

[11] K. Belashchenko, Phys. Rev. Lett. 2010, 105, 147204.

[12] N. Wu, X. He, A. Wysocki, U. Lanke, T. Komesu, K. Belashchenko, C. Binek, P. Dowben, Phys. Rev. Lett. 2011, 106, 087202.

[13] X. He, Y. Wang, Ni. Wu, A. N. Caruso, E. Vescovo, K. D. Belashchenko, P. A. Dowben, Ch. Binek, Nat. Mater. 2010, 9, 579.

[14] A. P. Malozemoff, Phys. Rev. B 1987, 35, 3697.

[15] L. Fallarino, A. Berger, C. Binek, Appl. Phys. Lett. 2014, 104, 022403.

[16] I. Schmid, A. A. Marioni, P. Kappenberger, S. Romer, M. Parlinska- Wojtan, H. J. Hug, O. Hellwig, M. J. Carey, E. E. Fullerton, Phys. Rev. Lett. 2010, 105, 197201.

[17] R. Sbiaa, M. Ranjbar, J Akerman, J. Appl. Phys. 2015, 117, 17C102 .

[18] A. W. Rushforth, P. C. Main, B. L. Gallagher, C. H. Marrows, B. J. Hickey, E. D. Dahlberg, P. Eames, J. Appl. Phys. 2001, 89, 7534.

[19] J. R. Barnes, S. J. O'Shea, M. E. Welland, J.-Y. Kim, J. E. Evetts, R. E. Somekh, J. Appl. Phys. 1994, 76, 2974.

[20] W. Peng, R. H. Victora, J. H. Judy, K. Gao, J. M. Sivertsen, J. Appl. Phys. 2000, 87,6358 .

[21] L. Wu, N. Honda, K. Ouchi, IEEE Trans. Magn. 1999, 35, 2775.

[22] R. Sbiaa, C. Z. Hua, S. N. Piramanayagam, R. Law, K. O. Aung, N. Thiyagarajah, J. Appl. Phys. 2009, 106, 023906.

[23] R. Sbiaa, Z. Bilin, M. Ranjbar, H. K. Tan, S. J. Wong, S. N. Piramanayagam, T. C. Chong, J. Appl. Phys. 2010, 107, 103901.

[24] L. Tryputen, F. Guo, F. Liu, T. N. A. Nguyen, M. S. Mohseni, S. Chung, Y. Fang, J. Akerman, R. D. McMichael, C. A. Ross, Phys. Rev. B 2015, 91, 014407.

[25] A. Berger, S. Mangin, J. McCord, O. Hellwig, E. E. Fullerton, Phys. Rev. B 2010, 82, 104423.

[26] Y. Shiratsuchi, S. Kawahara, H. Noutomi, R. Nakatani, IEEE Trans. Magn. 2010, 46, 1618 .

[27] J. Nogués, J. Sort, V. Langlais, V. Skumryev, S. Suriñach, J. S. Muñoz, M. D. Baró, Phys. Rep. 2005, 422, 65.

[28] P. Miltényi, M. Gierlings, M. Bamming, U. May, G. Güntherodt, J. Nogués, M. Gruyters, C. Leighton, I. K. Schuller, Appl. Phys. Lett. 1999, 75, 2304 .

[29] P. Borisov, W. Kleeman, J. Appl. Phys. 2011, 110, 033917.

[30] P. Barisov, A. Hochstrat, X. Chen, W. Kleemann, Phase Transition 2006, 79, 1123.

[31] K. O'Grady, L. E. Fernandez-Outon, G. Vallejo-Fernandez, J. Magn. Magn. Mater. 2010, 322, 883 .

[32] M. Rickart, A. Guedes, J. Ventura, J. B. Sousa, P. P. Freitas, J. Appl. Phys. 2005, 97, $10 \mathrm{~K} 110$.

[33] S. Soeya, T. Imagawa, K. Mitsuoka, S. Narishige, J. Appl. Phys. 1994, 76, 5356.

[34] E. L. Crow, Lognormal Distribution: Theory and Application, Marcel Dekker Inc, New York, USA 1998. 
[35] C.-H. Lai, T. J. Regan, R. L. White, J. Appl. Phys. 1997, 81, 3989.

[36] W. Zang, K. M. Krishnan, J. Appl. Phys. 2014, 115, $17 D 714$.

[37] V. Baltz, B. Rodmacq, A. Zarefy, L. Lechevallier, B. Dieny, Phys. Rev. B 2010, 81, 052404 .

[38] M. H. Kryder, E. C. Gage, T. W. McDaniel, W. A. Challenger, R. E. Rottmayer, G. Ju, Y.-T. Hsia, M. F. Erden, Proc. IEEE 2008, 96, 1810.

[39] V. Skumryev, S. Stoyanov, Y. Zhang, G. Hadjipanayis, D. Givord, J. Nogués, Nature 2003, 423, 850 .

[40] A. Scholl, F. Nolting, J. W. Seo, H. Ohldag, J. Stöhr, R. Raoux, J.-P. Locquet, J. Fompeyrine, Appl. Phys. Lett. 2004, 85, 4085.

[41] R. Morales, V. Vélez, O. Petracic, I. V. Roshchin, Z.-P. Li, X. Batlle, J. M. Alameda, I. K. Schuller, Appl. Phys. Lett. 2009, 95, 092503.

[42] W. A. Challener, C. Peng, A. V. Itagi, D. Karns, W. Peng, X. Yang, X. Zhu, N. J. Gokemeiher, Y.-T. Hsia, G. Ju, R. E. Rottmayer, M. A. Seigler, E. C. Gage, Nat. Photonics 2009, 3, 220 .

[43] T. Nozaki, M. Oida, T. Ashida, N. Shimomura, T. Shibata, M. Sahashi, Appl. Phys. Lett. 2014, 105, 212406.

[44] N. J. Gökemeijer, T. Ambrose, C. L. Chien, Phys. Rev. Lett. 1997, 79, 4270.

[45] U. Singh, S. Adenwalla, Nanotechnology 2015, 26, 255707.

[46] U. Singh, S. Adenwalla, Proc. SPIE 2013, 8813, 88131R .

Supporting Information follows ... 


\section{Supplemental document}

\section{Local Writing of Exchange Biased Domains in a Heterostructure of $\mathrm{Co} / \mathrm{Pd}$ pinned by Magnetoelectric Chromia}

Uday Singh, W. Echtenkamp, M. Street, Ch. Binek and S. Adenwalla*

\section{S1-Sample preparation}

The thin film sample of $\mathrm{Cr}_{2} \mathrm{O}_{3}$ was deposited on the c-plane of single-crystal $\alpha-\mathrm{Al}_{2} \mathrm{O}_{3}$ which was cleaned according to modified RCA cleaning protocols [1]. A pulsed KrF excimer laser with pulse energies of $150 \mathrm{~mJ}$ and a repetition rate of $5 \mathrm{~Hz}$ was used to ablate a pure $\mathrm{Cr}_{2} \mathrm{O}_{3}$ target, depositing $300 \mathrm{~nm}$ of (0001) textured $\mathrm{Cr}_{2} \mathrm{O}_{3}$ on the substrate. During deposition the substrate temperature was held at $700{ }^{\circ} \mathrm{C}$ and the pressure in the deposition chamber was $1.5 \times 10^{-}$ ${ }^{7}$ mbar. After this initial deposition, the temperature was reduced and the sample was transferred to a separate chamber for the FM film to be deposited. The temperature of the sample was raised to $800{ }^{\circ} \mathrm{C}$ for a duration of 6 hours just prior to deposition of the $\mathrm{Co} / \mathrm{Pd}$ multilayers to desorb any water molecules on the $\mathrm{Cr}_{2} \mathrm{O}_{3}$ surface which might have adsorbed while transferring from the PLD to $\mathrm{MBE}$ system. Next a $\operatorname{Pd}(0.5 \mathrm{~nm}) /[\mathrm{Co}(0.6 \mathrm{~nm}) / \operatorname{Pd}(1.0 \mathrm{~nm})] \times 3$ multilayer with perpendicular magnetic anisotropy was deposited by molecular beam epitaxy. During the deposition of the ferromagnetic multilayer the sample temperature was held at $300{ }^{\circ} \mathrm{C}$ and the pressure in the deposition chamber was between $6.5 \times 10^{-8}$ mbar and $1.1 \times 10^{-7}$ mbar.

[1] Dan Zhang, You Wang, and Yan Gan, Appl. Surf. Sci. 2013, 274, 405. 


\section{S2-Focused MOKE setup}

Spatially resolved exchange bias imaging was performed using two separate focused MOKE setups to acquire local hysteresis loops. Temperature control and scanning capabilities was established using a thermoelectric cooler mounted on a scanning stage

One focused MOKE apparatus [1-2], with a Gaussian beam spot of standard deviation 1.9um consisted of a $10 \mathrm{~mW}$ He-Ne laser beam, which passes through two polarizers. The first polarizer controls the intensity of the laser beam by changing the relative polarization angle with respect to the second polarizer. The second polarizer determines the polarization of the light used for the MOKE measurement. Prisms are used to reflect the polarized beam onto a focusing objective lens (Edmund $20 \mathrm{X}$ DIN achromatic). When the sample surface is at the focal plane of the objective lens, the laser beam is focused to a Gaussian spot of beam width (standard deviation) $\sigma=1.9$ um with an angle of incidence of $\sim 15$ degrees. The reflected beam from the sample is collimated using the same objective lens and is directed to a photodetector (Thorlabs DET 10A) after passing through an analyzer. The signal from the photodetector is measured using a nanovoltmeter (Keithley 2182A). With the sample in position, the analyzer and polarizer are crossed to minimize the photodetector signal. Hysteresis loops are measured with the analyzer rotated by 1 degree with respect to this minimum.

To obtain higher spatial resolution with tighter focusing, the second MOKE set up uses a laser beam from a solid state laser diode (laser diode (HL6501MG) mounted on a temperature controller (TCLDM9)) that is focused using a high power objective lens (Edmund 60X DIN achromatic NA 0.75 ). To enhance signal to noise, the laser intensity is modulated at $1201 \mathrm{~Hz}$ and the signal from the photodetector is measured using a lock-in (SRS830). As shown in figure S2- 
2, the laser beam is p-polarized and passes through a 50/50 beam splitter(BS). The vertical beam is incident on a high power objective lens (L) focusing the beam to a Gaussian spot of beam width $\sigma=0.8 \mathrm{um}$, with normal incidence on the sample surface. The reflected laser beam is collimated by the same objective lens and traverses the same path as the incident laser beam. After passing through the beam splitter and an analyzer (A), the reflected laser beam is detected by a photodetector connected to a lock-in amplifier operating at the laser modulation frequency. After minimization of the photodetector signal, the analyzer is rotated by 1 degree and the optical signal is measured at the input of the lock-in.

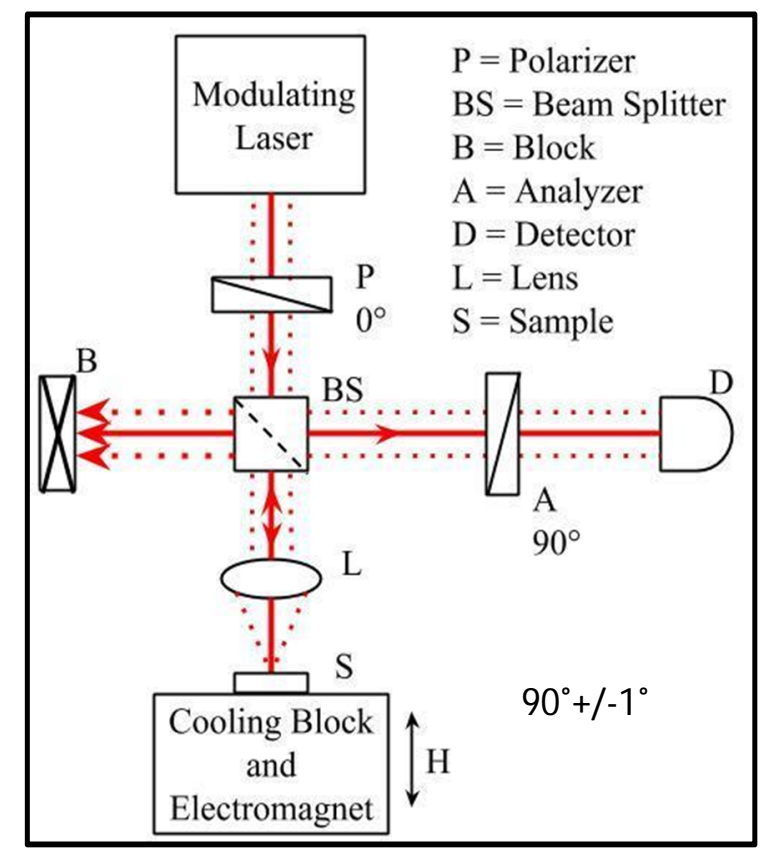

Figure S2-1: A sketch of the focused MOKE apparatus using the DIN 60x objective. The laser beam from a laser diode is focused to a Gaussian spot of standard deviation 0.8um, giving higher spatial resolution.

Temperature dependent measurements were performed by mounting the sample on an aluminum cooling unit. The sample was placed on a $0.5 \mathrm{~mm}$ thick copper plate and its temperature was maintained by controlling the current to the TEC using a Kepco bipolar power 
supply via a Labview program. This arrangement allowed for temperature control over a range of $267 \mathrm{~K}$ to $340 \mathrm{~K}$ with an accuracy of $0.01 \mathrm{~K}$. The cooling block also houses an electromagnet for application of out-of-plane magnetic fields. A small indentation in the cooling block and a hole in the thermoelectric cooler (P/N CH-109-1.4-1.5, TE Technology Inc.) allowed the electromagnet pole piece to be within $1 \mathrm{~mm}$ of the sample. The entire cooling block with the electromagnet was water cooled and the whole setup was mounted on a micrometer stage driven by a DC servo motor controlled by a Newport ESP 300 controller.

[1] Uday Singh and S. Adenwalla, Nanotechnology 2015, 26, 255707.

[2] Uday Singh and S. Adenwalla, Proc. SPIE 2013, 8813, Spintronics VI, 88131R. 


\section{S3-MFM scan}

MFM scans at room temperature showed the variation in the size, structure and density of FM domains structure for different magnetization states.

A: After Positive Saturation
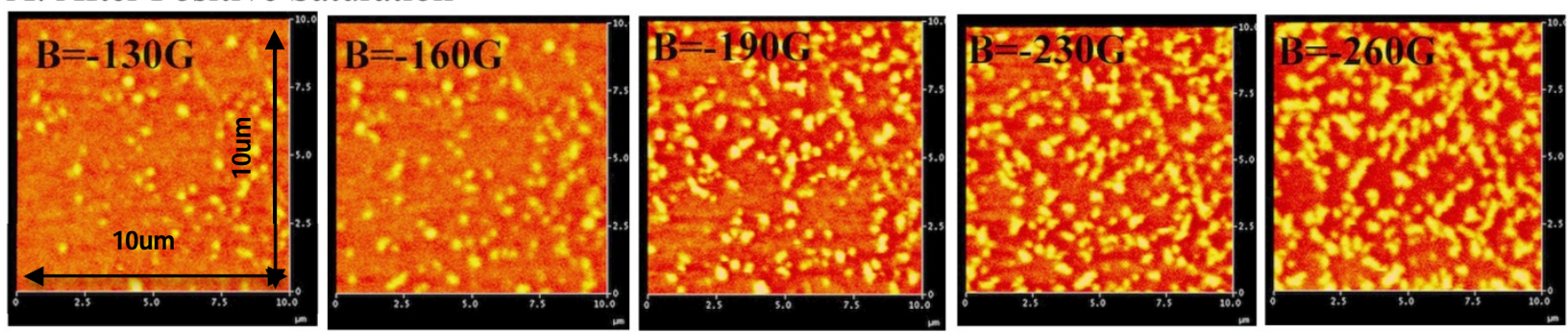

B: After Negative Saturation
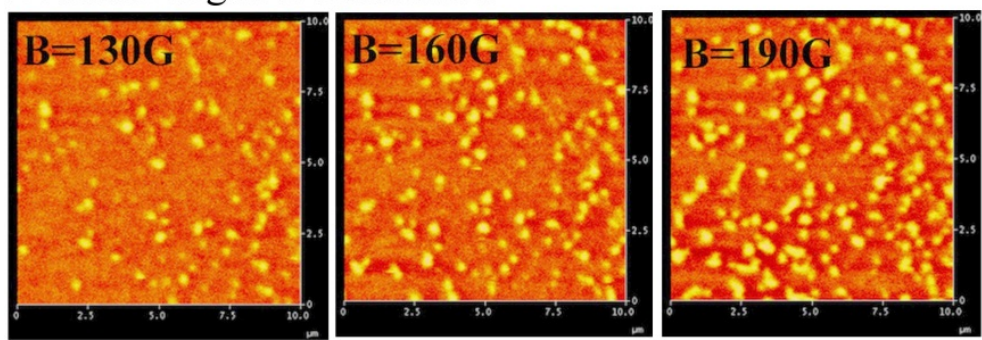

C: After Demagnetization

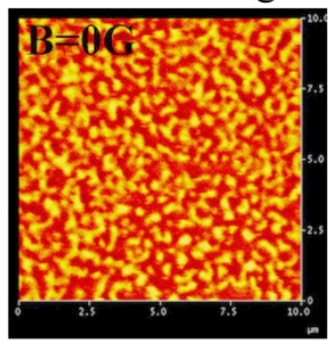

Figure S3-1: MFM scans (10umx 10um) of FM domains at room temperature ( 296K) in different magnetic fields after, A) positive saturation and B) negative saturation. The scans show the variations in the distribution of up and down domains at different magnetization states. C) MFM scan after ac demagnetization.

The distribution and sizes of minority domains were obtained by counting the area occupied by up and down domains, using ImageJ for the analysis of MFM images. The FM 
domain size distribution for the various field values is shown in figure S3-2, together with a fit to a lognormal distribution function. Domain sizes increase as the field varies from saturation to the coercive field, for both positive and negative saturation.
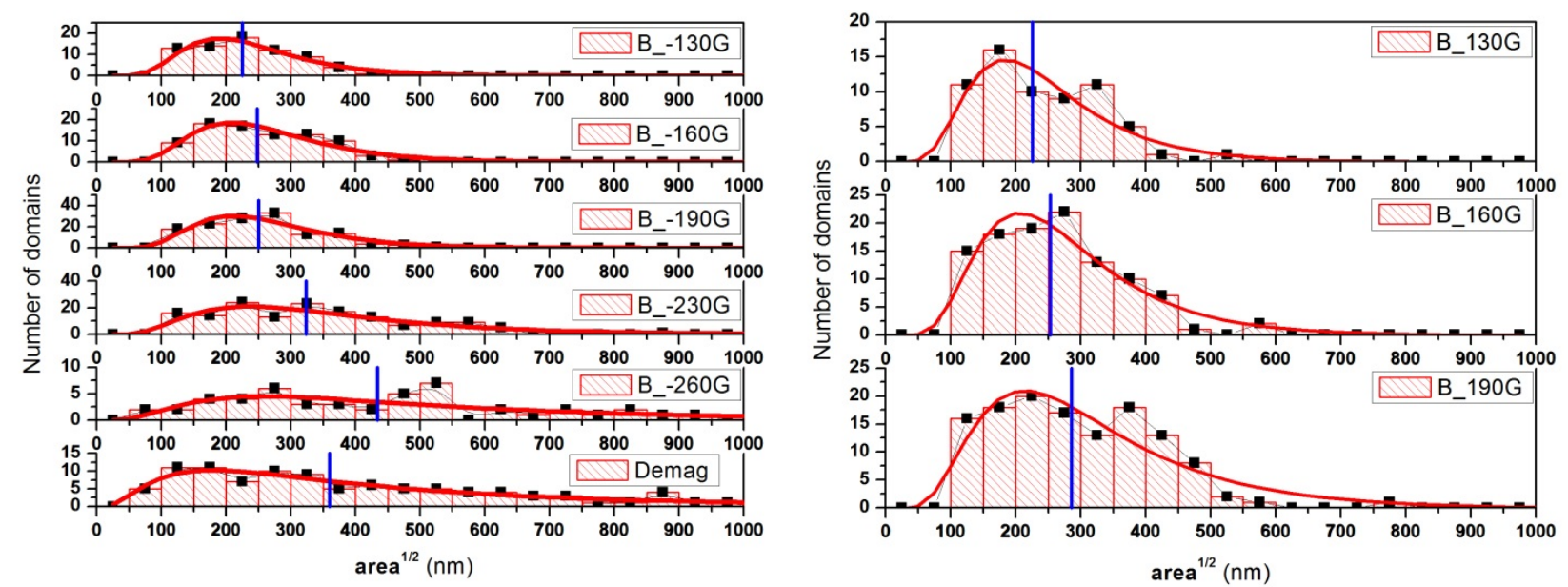

Figure S3-2: The size distribution of minority magnetic domains obtained using ImageJ from the MFM scans (fig. S3-1). The histograms are fit to a lognormal distribution and the blue line indicates the median of the distribution.

The net magnetization (figure S3-3) within the focused MOKE area may also be obtained from the MFM data., Each 10um X 10um MFM scan was sliced into sixteen 2.5um x 2.5um regions (equal to the step size used in the maps of exchange bias (figure 7(a))). Within each of these regions, the area occupied by up and down domains was found using ImageJ and the net magnetization calculated, resulting in histograms of the net magnetization. Note that the magnetization is not uniformly distributed even for the demagnetized sample, in which small domains might be expected to contribute a net zero magnetization on the $2.5 \mathrm{um}$ scale. This spatial variation of the magnetization on the scale of $2.5 \mathrm{um}$ is a major contributor to the large variation in exchange bias at low temperature (figure 7(b)). 


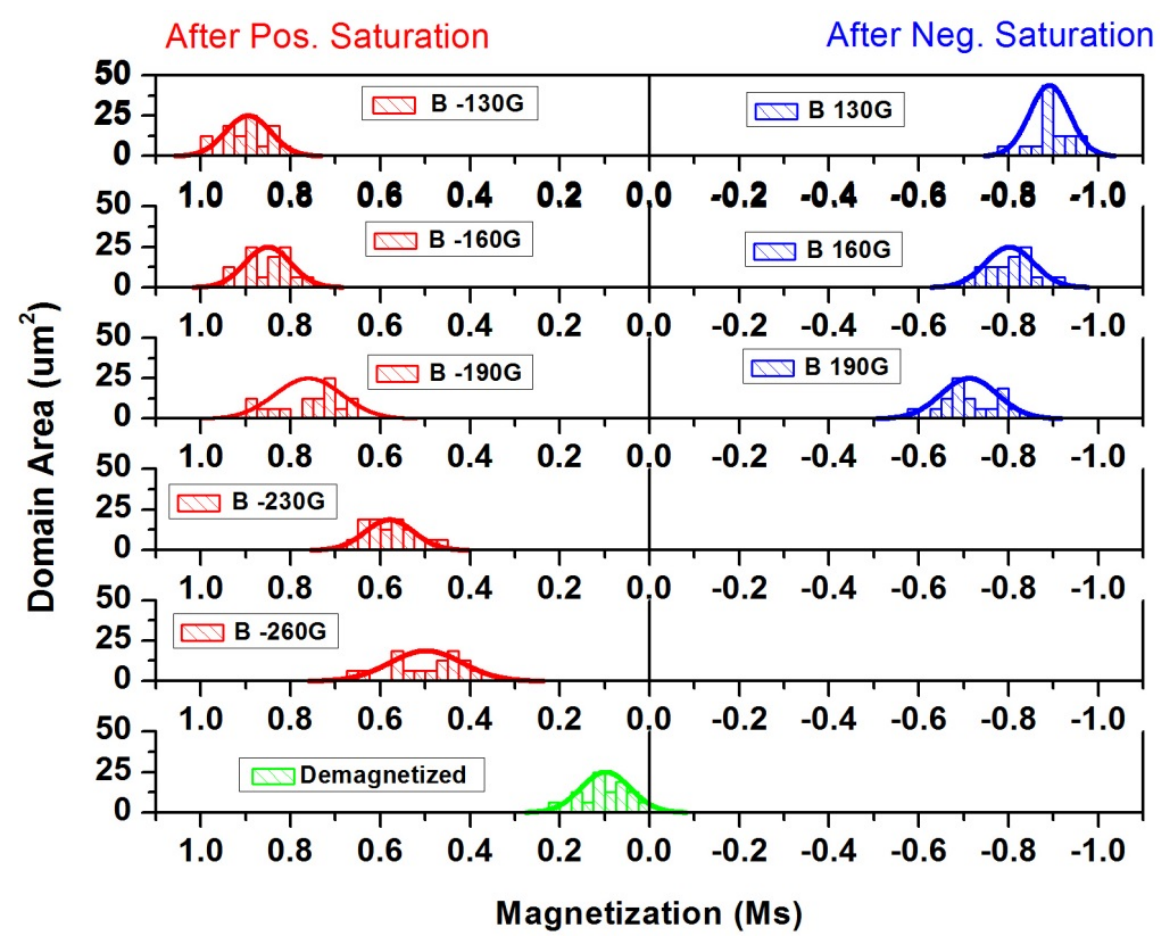

Figure S3-3: Histogram of the area occupied by FM domains at different field, showing the total area corresponding to a given magnetization, binned in units of $0.025 \mathrm{Ms}$, for a variety of applied fields.

\section{S4-Exchange bias map}

To understand the dependence of the exchange bias on the FM layer magnetization and temperature, the exchange bias was mapped for four different cooling conditions (M/Ms $= \pm 0.8$, $\mathrm{M} / \mathrm{Ms}=0(\mathrm{ZFC})$ and $\mathrm{M} / \mathrm{Ms}=0\left(\mathrm{FC}\right.$ at $\left.\left.\mathrm{H}_{\mathrm{c}}\right)\right)$. The magnetization was locked in at $313 \mathrm{~K}$, after which the sample temperature was lowered to $268 \mathrm{~K}$. Spatially resolved hysteresis loops were measured at $268 \mathrm{~K}$, mapping the exchange bias at a step size of $2.5 \mathrm{um} \times 2.5 \mathrm{um}$. The mapping was repeated for various temperatures, including temperatures below and above the blocking temperature of the system (290K). Figure S4-1 shows the exchange bias map at different temperatures under various cooling conditions. 


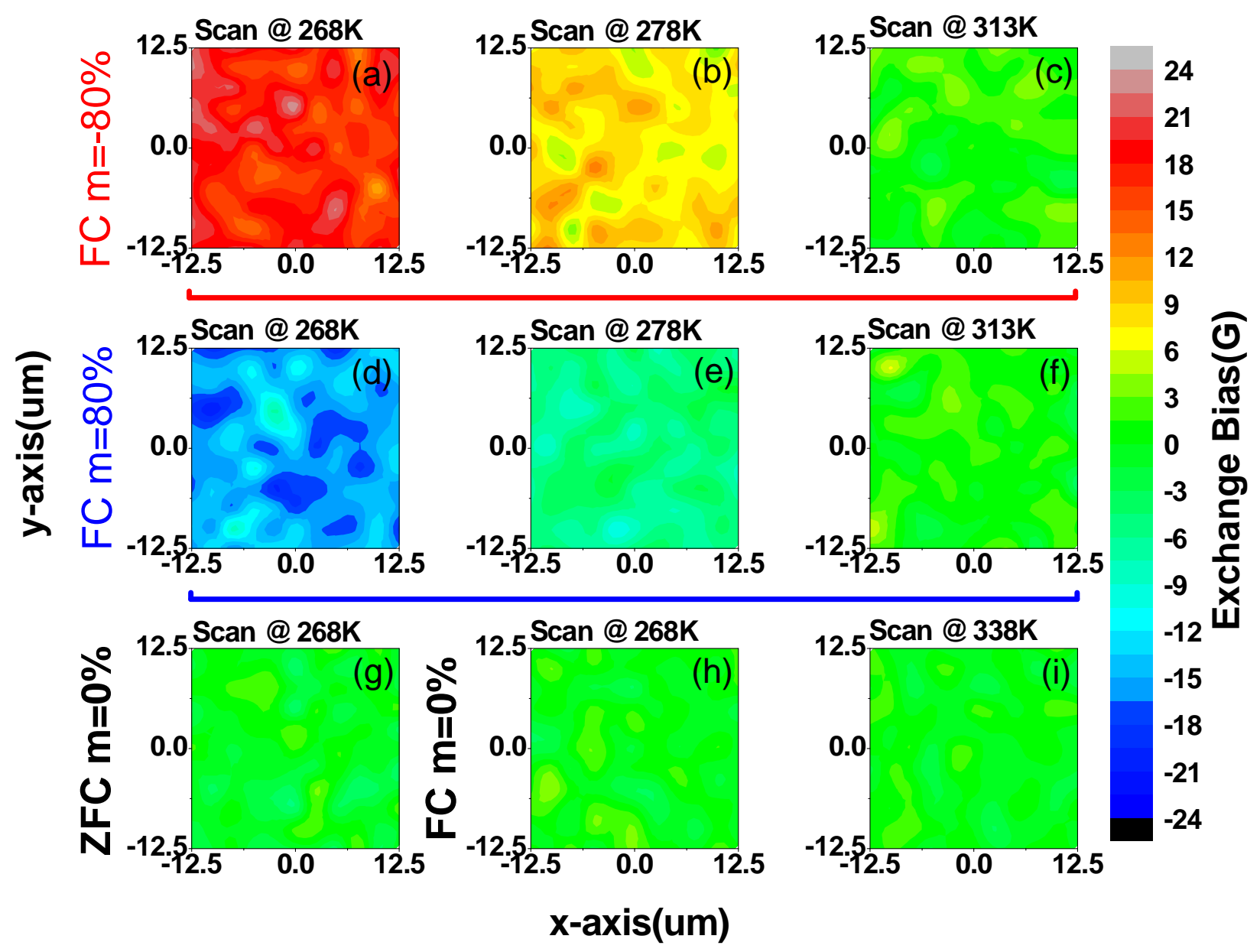

Figure S4-1: Spatial map of the exchange bias at different temperatures and under various cooling conditions, given by $\mathrm{m}=\mathrm{M} / \mathrm{M}_{\mathrm{s}}$.

To quantify the spatial variation in exchange bias, area histograms of the exchange bias with a bin size of $1 \mathrm{G}$ are shown in figure S4-2 for all temperatures and magnetic field conditions shown in figure S4-1. 

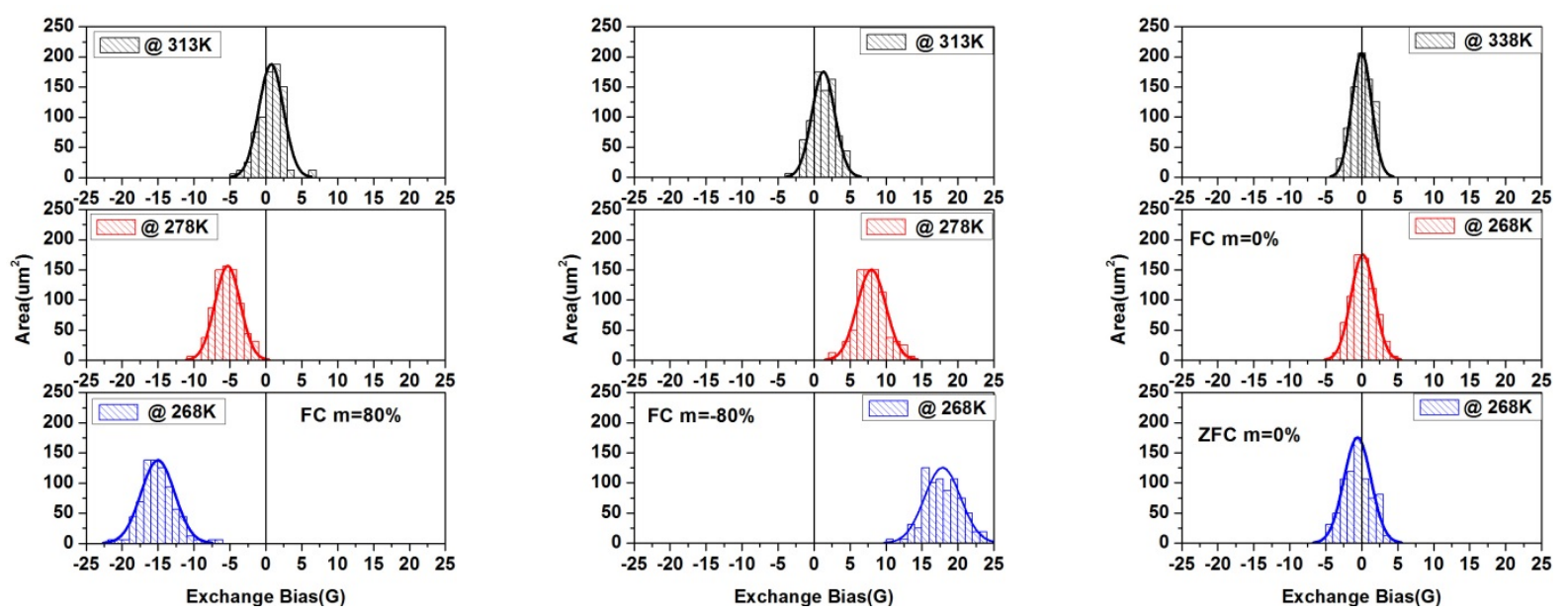

Figure S4-2: Histograms of the distribution of exchange bias obtained from the exchange bias area maps at different temperatures (while heating) for different magnetization cooling conditions $(\mathrm{M}= \pm 0.8 \mathrm{Ms}, \mathrm{M}=0(\mathrm{ZFC})$ and $\mathrm{M}=0(\mathrm{FC}))$. At low temperature $(268 \mathrm{~K})$, the mean value of $\mathrm{EB}$ is large and positive (negative) for cooling in $\mathrm{M}=-0.8 \mathrm{Ms}(+0.8 \mathrm{Ms})$, decreasing to zero exchange bias at high temperatures. For the other two magnetic cooling conditions $(\mathrm{M}=0(\mathrm{ZFC})$ and $\mathrm{M}=0(\mathrm{FC}))$, the exchange bias at low temperature $(268 \mathrm{~K})$ is nearly zero.

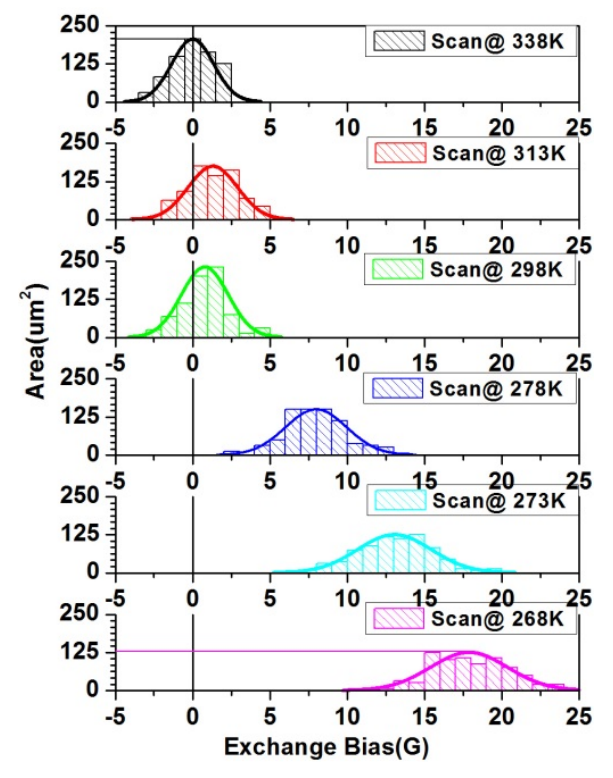

Figure S4-3: Detailed temperature dependence of the distribution of exchange bias for $\mathrm{M}=0.8 \mathrm{M}_{\mathrm{s}}$. At low temperature, the EB is large with a wide distribution. With increasing temperature, both the exchange bias and the width of the distribution decrease till the blocking temperature $(290 \mathrm{~K})$ and remain unchanged for all temperatures above it. 


\section{S8-Simulating temperature profile}

The 1D inhomogeneous heat equation for the laser heated surface is given by

$$
\frac{\partial T}{\partial t}=\alpha \nabla^{2} T+\beta f(x)
$$

where $\alpha$ is the thermal diffusivity of the system, $f(x)$ is the 1 dimensional profile of the heat source (in this case, the laser beam) and $\beta$ is the proportionality constant between the laser intensity and the rise in temperature. The value of $\beta$ depends upon the reflectivity, absorbance of the surface and the laser. In our experiments the intensity distribution of the focused laser beam is a Gaussian with a standard deviation $(\sigma)$ of 0.8 um incident on the sample surface, and is given by

$$
f(x)=e^{-\left(\frac{x^{2}}{2 \sigma^{2}}\right)}
$$

We have used the finite difference method [1] to solve the inhomogeneous heat equation and simulate the temperature profile of the laser heated surface. Using the numerical scheme of forward in time and center in space, the time derivative and second derivative in space becomes

$\frac{T(x, t+\Delta t)-T(x, t)}{\Delta t}=\alpha\left(\frac{T(x+\Delta x, t)-2 T(x, t)+T(x-\Delta x, t)}{(\Delta x)^{2}}\right)+\beta f(x)$

Discretizing the above equation we get,

$$
\frac{T_{i}^{n+1}-T_{i}^{n}}{\Delta t}=\alpha\left(\frac{T_{i+1}^{n}-2 T_{i}^{n}+T_{i-1}^{n}}{(\Delta x)^{2}}\right)+\beta f\left(x_{i}\right)
$$


And on simplifying this we get,

$$
T_{i}^{n+1}=T_{i}^{n}+\alpha \Delta t\left(\frac{T_{i+1}^{n}-2 T_{i}^{n}+T_{i-1}^{n}}{(\Delta x)^{2}}\right)+\beta \Delta t f\left(x_{i}\right)
$$

The convergence of the above heat equation is only possible if

$$
\frac{\alpha \Delta t}{(\Delta x)^{2}} \leq \frac{1}{2}
$$

Equation 5 was solved using a Matlab program, subject to the initial condition $\mathrm{T}(\mathrm{x}, 0)=0$ and zero temperature at the boundary.
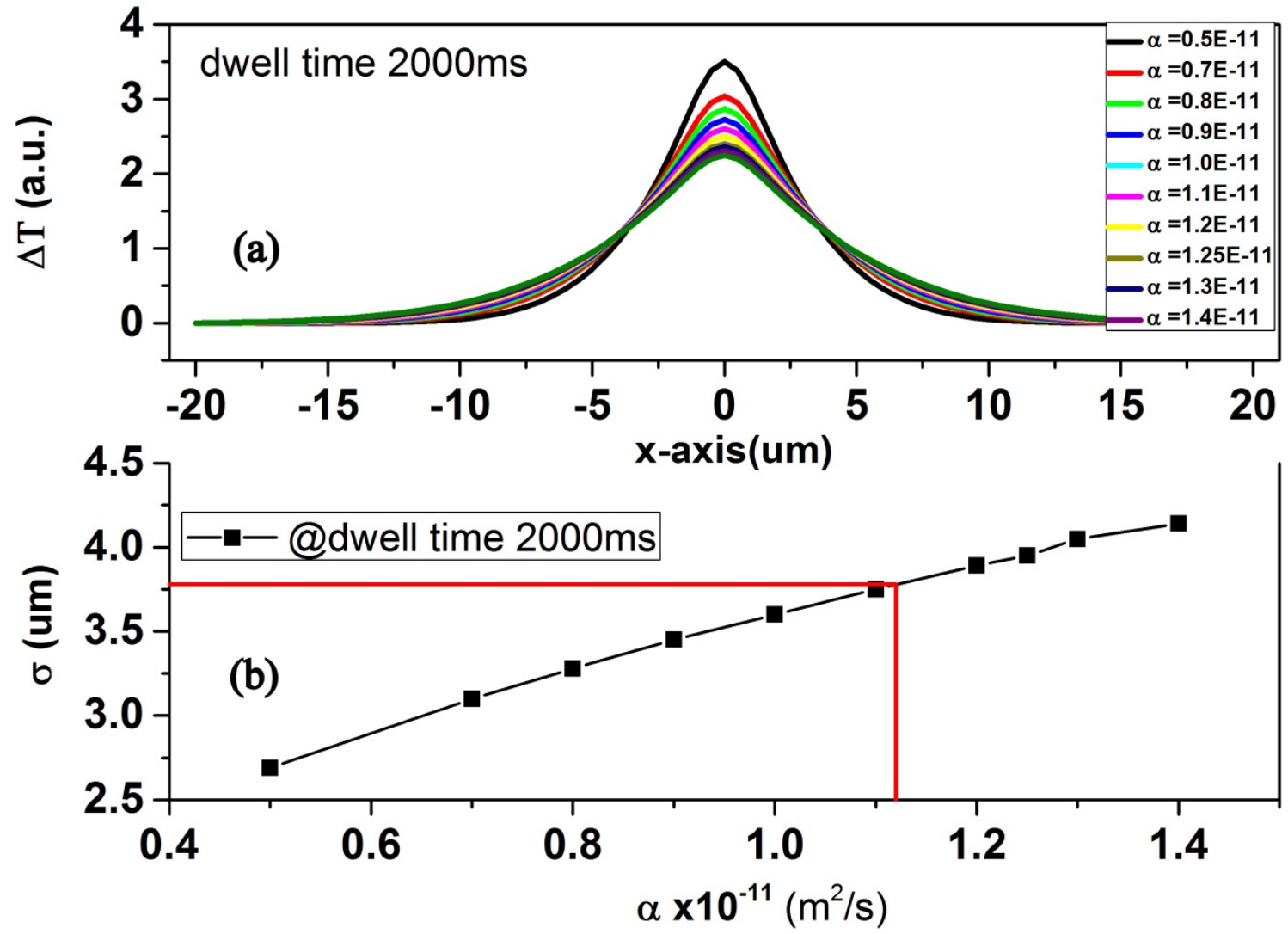

Figure S8: (a) Simulated temperature profile and (b) Gaussian width ( $\sigma$ ) for different values of $\alpha$. 
To account for the variation in the exchange bias profile at different writing speeds, we start by fitting the wider profile that arises from the lower writing speed. The laser beam advances in discrete 1 um steps; hence, for the lower writing speed of $0.5 \mathrm{um} / \mathrm{sec}$, for each step, it dwells on the surface for $2000 \mathrm{~ms}$. For the faster writing speed, $(2.5 \mathrm{um} / \mathrm{sec})$ the dwell time is $400 \mathrm{~ms}$. We first simulate the temperature profile for the $2000 \mathrm{~ms}$ dwell time. The step sizes in the equation are set to $\Delta \mathrm{t}=1 \mathrm{~ms}, \Delta \mathrm{x}=0.5 \mathrm{um}$, the parameter $\beta=1 \mathrm{E}-9 \mathrm{~K} / \mathrm{s}$, and the simulation is run for $2000 \mathrm{~ms} . \alpha$ is allowed to vary. This specific value of $\beta$ was chosen so as to have the rate of temperature change due to laser heating to be of the same order of magnitude as the rate of temperature change due to thermal diffusion. The simulated temperature profiles for a range of $\alpha$ values are shown in figure S8(a) and the width of the Gaussian temperature profile is extracted and plotted in figure $S 8(b)$, as a function of $\alpha$. Since our objective is to best fit the data, we choose that value of $\alpha\left(1.1 \mathrm{E}-11 \mathrm{~m}^{2} / \mathrm{s}\right)$ that matches the experimentally determined Gaussian profile (with a standard deviation of $3.78 \mathrm{um}$ ) of exchange bias written at a speed of $0.5 \mathrm{um} / \mathrm{s}$.

Note that the material constant $(\alpha)$ in our simulation is not equal to the thermal diffusivity of the material-rather it is scaled to satisfy the convergence condition (equation. (6)). Having obtained a "best-fit" value for $\alpha$, we simulate the temperature profile for the faster writing speed with dwell time of $400 \mathrm{~ms}$ (speed $2.5 \mathrm{um} / \mathrm{s}$ ). This shows excellent agreement with the experimentally obtained exchange bias profile, validating our argument that heat diffusion during writing is important in controlling the width of the exchange bias profile. 